\title{
Advances in Engineered Three-Dimensional (3D) Body Articulation Unit Models
}

\author{
Ying Chen ${ }^{1,2}$ \\ Ying Wang ${ }^{3,4}$ \\ Sheng-Chang Luo ${ }^{1,2}$ \\ Xiang Zheng ${ }^{1,2}$ \\ Ranjith Kumar Kankala (D) ${ }^{1,2}$ \\ Shi-Bin Wang ${ }^{1,2}$ \\ Ai-Zheng Chen (D) ${ }^{1,2}$ \\ 'Institute of Biomaterials and Tissue \\ Engineering, Huaqiao University, Xiamen, \\ 36I02I, Fujian, People's Republic of China; \\ ${ }^{2}$ Fujian Provincial Key Laboratory of \\ Biochemical Technology (Huaqiao \\ University), Xiamen, 361021, Fujian, \\ People's Republic of China; ${ }^{3}$ Affiliated \\ Dongguan Hospital, Southern Medical \\ University, Dongguan, 523059, Guangdong, \\ People's Republic of China; ${ }^{4}$ Guangdong \\ Provincial Key Laboratory of Shock and \\ Microcirculation, Guangzhou, 510080, \\ Guangdong, People's Republic of China
}

Correspondence: Ranjith Kumar Kankala; Ai-Zheng Chen

Institute of Biomaterials and Tissue

Engineering, Huaqiao University, Xiamen,

36I02I, People's Republic of China

Email azchen@hqu.edu.cn;

ranjithkankala@hqu.edu.cn

\begin{abstract}
Indeed, the body articulation units, commonly referred to as body joints, play significant roles in the musculoskeletal system, enabling body flexibility. Nevertheless, these articulation units suffer from several pathological conditions, such as osteoarthritis (OA), rheumatoid arthritis (RA), ankylosing spondylitis, gout, and psoriatic arthritis. There exist several treatment modalities based on the utilization of anti-inflammatory and analgesic drugs, which can reduce or control the pathophysiological symptoms. Despite the success, these treatment modalities suffer from major shortcomings of enormous cost and poor recovery, limiting their applicability and requiring promising strategies. To address these limitations, several engineering strategies have been emerged as promising solutions in fabricating the body articulation as unit models towards local articulation repair for tissue regeneration and high-throughput screening for drug development. In this article, we present challenges related to the selection of biomaterials (natural and synthetic sources), construction of 3D articulation models (scaffold-free, scaffold-based, and organ-on-a-chip), architectural designs (microfluidics, bioprinting, electrospinning, and biomineralization), and the type of culture conditions (growth factors and active peptides). Then, we emphasize the applicability of these articulation units for emerging biomedical applications of drug screening and tissue repair/regeneration. In conclusion, we put forward the challenges and difficulties for the further clinical application of the in vitro 3D articulation unit models in terms of the long-term high activity of the models.
\end{abstract}

Keywords: 3D models, articulation disease, drug screening, bioprinting, tissue regeneration

\section{Introduction}

Indeed, the body articulation units, commonly referred to as joints (both movable and immovable), are the connections between two bones in the musculoskeletal system, enabling body flexibility and mobility. In general, these articulation units anatomically comprise different components, such as bone, ligaments, cartilage, tendons, bursas, synovial fluid, membrane, and meniscus. Several pathological conditions include osteoarthritis (OA), rheumatoid arthritis (RA), ankylosing spondylitis, gout, and psoriatic arthritis. Among them, arthritis is a common, chronic, and degenerative disease due to the obsessive changes in articular cartilage, joint capsule, and meniscus, manifested by inflammation, pain, stiffness, infectious degradation, and trauma. ${ }^{1,2}$ A variety of human cancers from prostate, breast, and lung tends to transfer to the bone articulation in later stages, resulting in further complications. ${ }^{3,4}$ Notably, about $10 \%$ of men and $18 \%$ of women over 60 years of age suffer from arthritis due to autoimmune reactions, infections, metabolic disorders, trauma, and degenerative diseases. ${ }^{3,5}$ Several treatment methods include 


\section{Graphical Abstract}

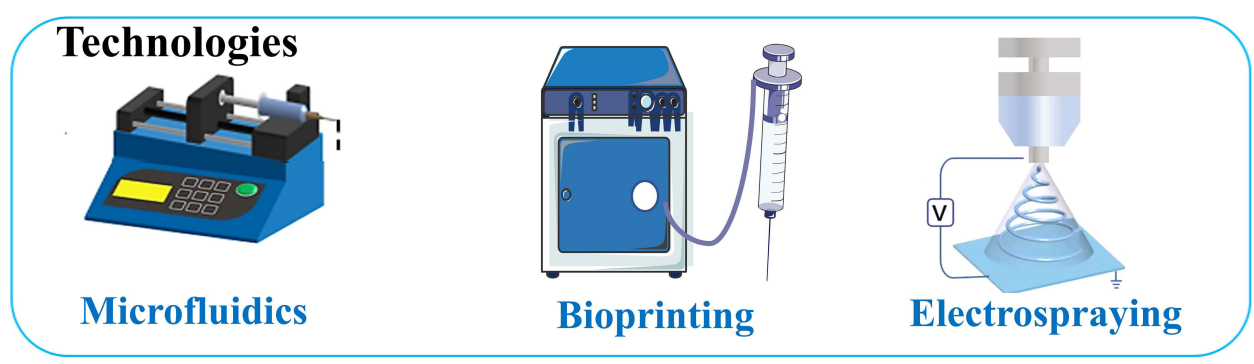

\section{Architectural designs}

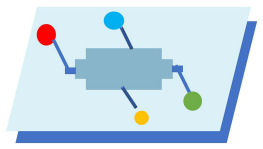

Organ-on-chips

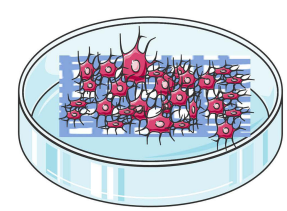

Composite scaffolds

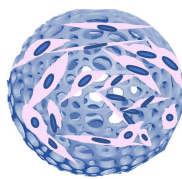

Porous microsphere

\section{Applications}

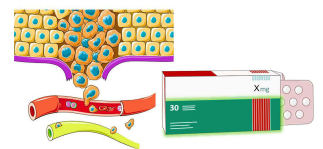

Drug screening

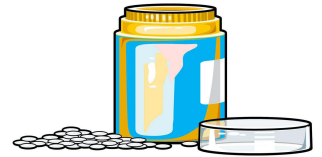

Drug development

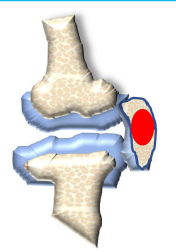

Tissue regeneration surgical procedures and the utilization of traditional steroids and non-steroidal anti-inflammatory drugs (NSAIDs). On the one hand, the surgical treatments are often limited due to their huge cost and poor recovery. On the other hand, the utilization of chemotherapeutic drugs and minimally invasive surgeries are often adopted to cure diseases in articulation units due to high security and low cost, which, however, lack specificity.

Apart from the conventional therapeutic strategies, engineering of the joints as unit models has been emerged as a promising solution towards addressing the shortcomings of the aforementioned conventional therapies. In this regard, the fabrication of the articulation units at the microscale provides not only the detailed exploration of complex joint diseases within the unit and at the interface between different units but also offers excellent prospects for the precise treatment of articulation diseases. These 3D models can simulate various physiological structures of articulation units, which can be applied for surgical replacement, tissue regeneration, and drug screening. In this framework, different architectural designs include porous microspheres (PMs), composite structures, and organ-onchips, which are of specific interest for various biomedical applications, such as drug screening towards drug development, and tissue regeneration. However, there still exist many limitations in the clinical application of 3D models. For example, 3D bioprinting technology is not yet fully capable of producing functional substitutes equivalent to the human vascular system. In this regard, the survival of the designed joint unit substitutes cannot survive for a long time due to the lack of the corresponding vascular system. Moreover, it is impossible to prepare a fine structural network to replace the intraosseous capillary network due to a high bone density and covered capillary network. In addition, the application of 3D models faces immunological challenges, usually resulting in the damaged matrix and invigorating cells. Thereby, depriving new tissues of homeostasis, remodeling, and integration hinders the further development for their application in regenerative medicine. In this review, we emphasize the selection of 
materials (natural materials, dECM, and synthetic materials) and biologically active substances (BMP-2, OGP, and some other active proteins) in joint unit tissue engineering. Further, various micro-tissue engineering strategies (microfluidics, bioprinting, electrospinning, biomineralization) employed in the fabrication of these unit models are explained, highlighting their pros and cons and effects of various processing parameters. Finally, we systematically emphasize the aspects of engineered 3D joint models for various biomedical applications of drug screening and tissue regeneration (Table 1).

\section{Construction of 3D Articulation Models}

The development of 3D models has garnered enormous attentiveness towards the treatment of articulation-based diseases and other multiple biomedical applications, such as drug screening and articulation repair. These 3D models can be precisely designed by harboring cells of interest (for instance, tumor cells and vascular cells) to develop a highly functionalized biomimetic tissue with appropriate vasculature for gaseous exchange and waste removal. In addition, various required signal molecules can be incorporated with controlled release to guide tumor cell metastasis. Importantly, these simulating articulation disease models can be used for screening of chemotherapeutic drugs for various articulation-based diseases. In general, articulation diseases often cause local tissue damage, in which cartilage defects are predominant. In view of engineering these models units with unique structures, these 3D cell adhesion scaffolds can be embedded with chemotherapeutic drugs or cell growth factors to further promote articulation repair. In this section, we explicitly introduce various fabricated designs for the construction of 3D cell adhesion scaffolds, including scaffold-free, scaffold-based, and organ-on-a-chip.

\section{Scaffold-Free Systems}

The scaffold-free cellularized system is a classic model composed of suspended cells with different proliferation rates. The outermost layer of cells offers high proliferation efficacy due to excessive nutrient supply, while the inner compact cell mass possesses relatively lesser proliferating efficacy due to the lack of sufficient nutrient supply. ${ }^{6-8}$ The spatial arrangement of cells in the scaffold-free systems simulates the internal physiological structure of articulation tumors. $^{9-11}$ These considerations have substantially made them suitable for basic research on physiological metabolism, tumor biology, and toxicology, as well as fabricating cellularized scaffolds. The cellular spheroids simulate a tumor mass and invade the surrounding matrix to form an invasive branch. By measuring the area of the spheroid and the length of invasion, the ability of each cell type to invade different cells can be evaluated (Figure 1). ${ }^{12}$ By harboring different types of cells and intervening with them to simulate the microenvironment of specific diseases, these models are cheaper and more reproducible than animal models. Briefly, the 3D cellular constructs are generally fabricated in a facile way, in which the centrifuged cells are initially stored in conical tubes or multi-well plates in pellets by centrifugation, forming a spherical culture system. Compared with monolayer and typical culture methods, suspension droplet culture promotes the aggregation of spheroids in the form of cells and improves the expression of Sox 9 mRNA. It was also shown that the suspension drop culture caused an increase in proteoglycan 4 (Prd4) mRNA levels, thereby providing lubrication to articulation. ${ }^{13}$ In another instance, the experimental results showed that the expressions of Sox 9 and Col2a1, as well as aggrecan mRNA in cartilage culture, significantly improved the ability of sediment culture cells. ${ }^{14,15}$ In addition to suspension drop culture of cell alone, some cellularized spheres embedded in agar and collagen have been fabricated, in which these $3 \mathrm{D}$ models further simulated the physiological interactions, metabolism, growth, and metastasis of cells, ${ }^{16}$ for instance, breast carcinoma $^{12}$ and lung cancer. ${ }^{17}$

\section{Scaffold-Based Systems}

Indeed, the tissue engineering field aims at fabricating the biomimetic constructs to simulate the anatomical and physiological features of tissue microenvironment for diverse biomedical applications, such as biodegradable hydrogels and polymeric microarchitectures. These scaffolding systems can be constructed to mimic the 3D structure of articulation by designing various materials with adjustable porosity, permeability, and surface chemistry. Several efforts have been dedicated to fabricating various biomimetic scaffolding systems towards treating bone joint disorders. For example, the cell type and matrix between the two connecting parts of the joint (cartilage and subchondral bone) were simulated by two well-combined and adjustable-shaped hydrogels to guide the duration of the inducer and the specific release of the defect site. ${ }^{18}$ Then, the sitespecific stem cells differentiated into cartilage and osteoblasts for joint repair. This strategy opened up a new 
Table I Examples of Various Cellularized Polymeric Architectural Designs Fabricated Using Various Microengineering Strategies for Medical Applications

\begin{tabular}{|c|c|c|c|c|c|}
\hline 3D Models & Materials & $\begin{array}{l}\text { Microengineering } \\
\text { Strategies }\end{array}$ & Cell Type & Applications & References \\
\hline \multirow{2}{*}{$\begin{array}{l}\text { Scaffold- } \\
\text { free }\end{array}$} & Matrigel & Drop culture & BMSCs & Articular cartilage repair & {$[9-11]$} \\
\hline & Alginate & Embedding sphere & Chondrocytes & OA model & {$[65]$} \\
\hline \multirow{20}{*}{$\begin{array}{l}\text { Scaffold- } \\
\text { based }\end{array}$} & PU-PCL/dECM & 3D cell-printing & BMSCs & Meniscus regeneration & {$[76]$} \\
\hline & Hydrogels & lonic crosslinking/Bioprinting & BMSCs & Articular cartilage repair & {$[153]$} \\
\hline & PEG/GelMA & Photo-crosslinking/Bioprinting & BMSCs & Articular cartilage repair & {$[154]$} \\
\hline & PEG/Hydrogels & Electrowritten & BMSCs & Articular cartilage repair & {$[113]$} \\
\hline & ECM/GelMA & 3D Printing & BMSCs & OA therapeutics & {$[155]$} \\
\hline & PLGA/TCP & Bioprinting & $\mathrm{BMSCs} / \mathrm{ECs}$ & Repair bone defects & {$[156]$} \\
\hline & HAP/PLLA & 3D printing & $\mathrm{OB} / \mathrm{ECs}$ & Repair bone defects & {$[157]$} \\
\hline & Silk fibroin & Photo-crosslinking/Bioprinting & BMSCs & Articular cartilage repair & [158] \\
\hline & Alginate & Suspension bioprinting & Chondrocytes & Simulate cartilage's pericellular matrix & [116] \\
\hline & Chitosan & Bioprinting & MCTS & Multi-tumor articulation metastasis model & {$[82]$} \\
\hline & GelMA/HA & Photo-crosslinking/Bioprinting & MFC & Repair damaged meniscal & [159] \\
\hline & Silk fibroin & Freeze-dry/Bioprinting & BMSCs & Cartilage regeneration & {$[115]$} \\
\hline & Chitosan/silk fibroin & Bioprinting & Chondrocytes & Cell proliferation and migration model & {$[85,115,160]$} \\
\hline & Gelatin/Collagen & Biomimetic mineralization & OB/BMSCs & Osteogenesis regeneration & [63] \\
\hline & PLGA/GelMA/dECM/ & $\begin{array}{l}\text { Co-axial microfluidics Photo- } \\
\text { crosslinking }\end{array}$ & $\mathrm{OB} / \mathrm{PC}$ & Prostate cancer Articulation bone metastasis & {$[55]$} \\
\hline & Collagen/PLGA/GelMA & $\begin{array}{l}\text { Coaxial microfluidics/Photo- } \\
\text { crosslinking }\end{array}$ & OB/BMSCs & Bone regeneration & {$[55,161]$} \\
\hline & PEG-based Collagen & Electrospinning & Bone cancer cells & Simulating bone cancer & {$[96]$} \\
\hline & $\begin{array}{l}\text { ZIF-8 (MOF)/PEG-based } \\
\text { hydrogels }\end{array}$ & Electrospinning & BMSCs & Simulated artificial periosteum & {$[128]$} \\
\hline & GelMA/ Collagen & Bioprinting & BMSCs & Articulation osteochondral repair & {$[83,128]$} \\
\hline & $\begin{array}{l}\text { GelMA/dECM-ZIF-8 } \\
\text { (MOF) }\end{array}$ & Bioprinting & BMSCs & Articulation osteogenesis repair & {$[123]$} \\
\hline \multirow{5}{*}{$\begin{array}{l}\text { Organ-on } \\
\text {-a-chip }\end{array}$} & Collagen/GelMA & Microfluidics & BMSCs & Organ model for drug screening & [35] \\
\hline & $\mathrm{PCL}$ & Microfluidics & $\begin{array}{l}\text { Breast cancer cell/ } \\
\text { BMSCs }\end{array}$ & Breast cancer metastases to articulation bone & {$[162]$} \\
\hline & PDMS & Microfluidics & $\begin{array}{l}\text { Breast cancer cell/ } \\
\text { BMSCs }\end{array}$ & Breast cancer metastases to articulation bone & {$[64]$} \\
\hline & PCL/PDMS & Microfluidics & OB/BMSCs & $\begin{array}{l}\text { Study the gene expression profile in } \\
\text { osteoarthritis. }\end{array}$ & {$[42]$} \\
\hline & PDMS & Microfluidics & Chondrocytes & $\begin{array}{l}\text { Study the characteristics of articulation and } \\
\text { react to injuries }\end{array}$ & [163] \\
\hline
\end{tabular}

Abbreviations: PU, polyurethane; PCL, polycaprolactone; dECM, decellularized extracellular matrix; MCTS, multicellular tumor spheroids; MFC, meniscal fibrochondrocyte; OA, osteoarthritis; BMSCs, bone mesenchymal stem cells; PC, prostate cancer cells; PEG, polyethylene glycol; GelMA, methacrylate gelatin; PLGA, Poly(lactic-coglycolic acid); HA, hyaluronic acid; MOF, metal-organic framework; PDMS, polydimethylsiloxane; TCP, tricalcium phosphate; Ecs, endothelial cells; HAP, incorporation of hydroxyapatite; PLLA, poly-L-lactic acid; OB, osteoblast. 


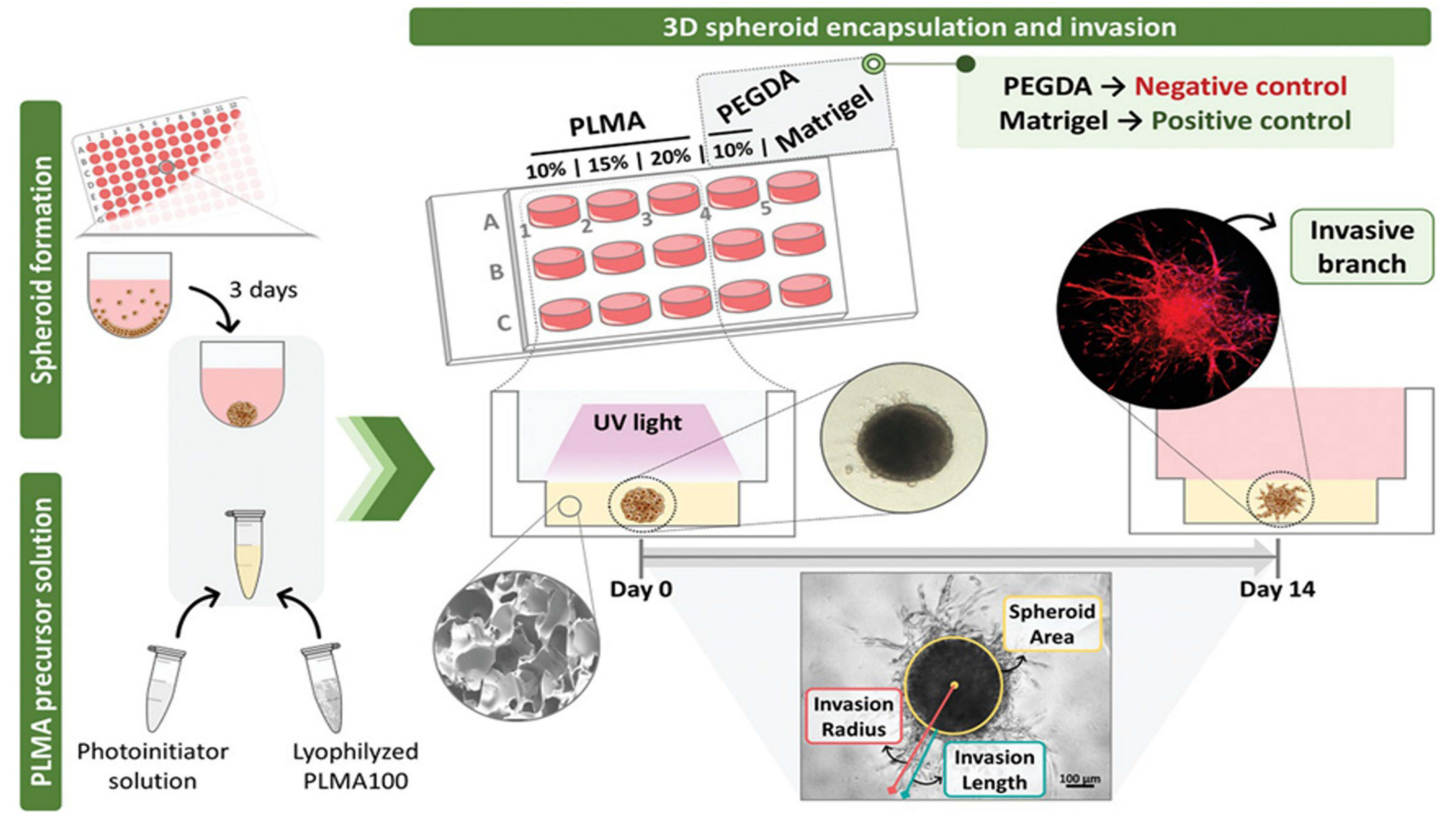

Figure I Schematics of 3D spheroids encapsulation to study cell spheroid invasiveness. Spheroids are formed into round-bottom ultralow attachment plates during $3 \mathrm{~d}$. The PLMA precursor solution is obtained solubilizing lyophilized PLMA in a photoinitiator solution for UV photopolymerization. Generated spheroids are encapsulated into PLMA hydrogels at three different concentrations (10, 15, and 20\% (w/v)), poly(ethylene glycol) diacrylate (PEGDA) at 10\% (w/v), and Matrigel. Over the time of culture, the encapsulated cells start to invade the surrounding ECM-mimicking matrix, developing the named invasive branches. Throughout the $14 \mathrm{~d}$ of culture, the spheroid area and invasion length are measured to evaluate the invasiveness ability of each cell type into the different biomaterials and stiffnesses. Reproduced with permission from Monteiro CF, Santos SC, Custódio CA, et al. Human plateletlysates-based hydrogels: a novel personalized 3D platform forspheroid invasion assessment. Adv Sci. 2020;7(7): 1902398. (C) 2020 The Authors. Published by WILEY-VCH Verlag GmbH \& Co. KGaA, Weinheim. This is an open access article distributed under the terms of the Creative Commons CC BY license. ${ }^{12}$

chemical method for biomaterials with stage-specific drug release for tissue repair. The hydrogel was fabricated by concurrently polymerizing two different layers using a facile one-pot synthesis method, resulting in the upper cartilage based on gelatin methacryloyl and lower subchondral bone-based on polydopamine hydrogels. The in situ mineralization of hydroxyapatite by polydopamine occurred in the lower layer to simulate the structural features of subchondral bone. Notably, the double-layer hydrogel exhibited excellent mechanical properties in terms of the combined effects of covalent and non-covalent linkages and the nano-reinforcement of mineralized hydroxyapatite. ${ }^{19}$ In addition to designing the optimal biomimetic scaffolds, it is essential to employ various inflammatory factors in exploring the pathological mechanism and treating the common joint disease, arthritis. ${ }^{20-23}$ This section focuses on a brief emphasis on the scaffolding system containing a variety of inflammatory factors that could help in understanding the pathophysiological mechanisms of arthritis towards drug screening. Fibroblast-like synoviocyte (FLS) is a crucial medium driven by articulation damage and inflammatory processes in arthritis. In addition to FLS, several reports indicated that the pro-inflammatory cytokines (for instance, tumor necrosis factor-alpha, TNF- $\alpha$, and interferon-gamma, IFN- $\gamma$ ) influenced the remodeling of articulation interstitial tissue. ${ }^{24}$ IFN invasive potential promoted the FLS gamma through Janus activation kinase (JAK), while TNF induced FLS apparent gathered themselves together, suggesting that these two factors affected the synovial tissue remodeling. The expression of IFN mediated inflammatory cascade reaction is the most important regulatory factor, confirmed by the TNF-mediated articulation model. ${ }^{25} \mathrm{~A} 3 \mathrm{D}$ synovial model made from a suspension of human primary synovial cells or a mixture of primary fibroblast-like synovial cells and CD14(+) monocytes were analyzed by immunohistochemical staining and flow cytometry. The composition of mature micelles reflected the naive synovium in the body. Exposing the micelles to the TNF- $\alpha$ results in the amplified expression and generation of pro-inflammatory cytokines, leading to membrane proliferation, which mimics the affected synovium in rheumatoid arthritis. Moreover, the exposure of the micelles to transforming growth factor $\beta$ (TGF- $\beta$ ) resulted in fibrosis-like changes, such as augmented $\alpha$-smooth muscle actin ( $\alpha$-SMA) and amplified expressions of fibrosis-related genes, COL1A1 and PLOD2. ${ }^{26}$ Remarkably, the macrophages in the micelles showed 
phenotypic plasticity, because persistent TNF- $\alpha$ or TGF- $\beta$ stimulation intensely reduced the incidence of CD163 M2like macrophages. Moreover, these consequences demonstrated the plasticity of micelles as a synovial model for studying the pathology of RA and OA, suggesting that the synovial lining micelle system could be an excellent substitute for drug screening. ${ }^{27}$

As mentioned earlier, the articular cartilage is one of the essential components of an articulation unit, which is commonly used for exploring the pathophysiological changes and treatment efficacy in articulation diseases. The human cartilage is mostly translucent avascular tissue with a relatively small number of cells. ${ }^{28}$ In general, the articulation cartilage consists of different layers (surface cut, excessive, radial), which can absorb mechanical load and force within the articulation unit. Notably, the cartilage cells are the only group of ECM constituents that produce and maintain a high degree of organization, consisting mainly of Type I, Type IX and Type XI. The inflammatory process is activated when a $3 \mathrm{D}$ tubular tissue model based on alginate is stimulated with the supernatant of RA synovial fibroblasts, leading to accelerated catabolism. ${ }^{29}$ Bold and coworkers developed an in vitro 3D cartilage model of RA for high-throughput drug screening through interactive co-cultivation of porcine cartilage and RA synovial fibroblast cell line. Introducing cell seeding automation and evaluation of cartilagespecific ECM formation improved the cell culture quality. ${ }^{30}$

\section{Organ-on-a-Chip}

Microfluidic technology offers several advantages of highspeed, parallel collection, and analysis of individual biological information. It is a crucial processing platform in modern biological sciences, providing a reliable technical support and operation platform for life research. ${ }^{31-34}$ This approach enables microfluidic chip-scale integration, micro-scale heat and mass transfer effects, controllable fluid flows, and similar bionic space microstructures. The resultant end products of the microfluidic chip technology can be applied to disease diagnosis and treatment by obtaining disease sample cells from lesion tissue and expanding them in vitro through specific culture conditions. Furthermore, the patient's exosomes can be collected to achieve individualized and precise disease diagnosis and drug screening. ${ }^{35}$

In general, articulation is a multi-tissue organ with different kinds of cells, in which the joint-on-A-chip can be generated by inoculating cells of interest, for instance, chondrocytes, into the chip and cultured for two weeks. The articulation units contain various cell types, such as stem cells, osteoblasts, osteoclasts, chondrocytes, and mature immune cells. ${ }^{36-38}$ Bone mesenchymal stem cells (BMSCs) are primarily used for articulation models owing to their differentiation ability into various cells. ${ }^{36,39}$ Osteoblasts and osteoclasts work together to maintain the dynamic balance of bones in osteoblasts. In this vein, the articular cartilage is an elastic weight-bearing structure that constitutes the articulation surface of a movable articulation. It should be noted that the articulation disease often leads to degeneration of articular cartilage. ${ }^{40}$ For instance, in synovial articulation, cells are subjected to high mechanical stress, which is through weight-bearing and the shear force generated by the movement of synovial fluid during exercise. ${ }^{41}$ In a case, after repeated pressure, the articular cartilage-on-the-chip was artificially overloaded, resulting in the appearance of arthritis symptoms. The genomic analysis results showed that the chip could simulate the cartilage tissue of articulation inflammation for OA treatment. ${ }^{42}$ In this work, an animal-free in vitro OA model was established by mixing in inflammatory cytokines, mimicking the essential characteristics of natural cartilage, and responding to biochemical damage. The appearance of the chip showed six individually addressable circular chambers, which could be realized by the experiment of culturing various types of cells to construct micro-tissues. A PDMS plate with a glass slide as the top layer and a microstructure as the bottom layer was fabricated, in which the hydrogel with cells could be loaded in the top chamber through the tip of a pipette, exploring the pathophysiology of $\mathrm{OA}$ in human and veterinary patients provides a new foundation. ${ }^{43}$ By interconnecting bioreactors with other compressed bioreactors, combinatorial screening of 3D cell behavior was performed (Figure 2A). The authors employed the changes in the diameter to control the pressure to simulate the compression strain experiment and data (Figure 2B-D). Moreover, the altered parameters and their effects on the proliferation and osteogenic differentiation of human mesenchymal stem cells (hMSCs) in 3D gelatin methacryloyl (GelMA hydrogel) were studied (Figure 2E and F). The multi-chamber structure could simulate the extent of articulation damage when the articulation were subjected to different sizes, directions, and types of pressures. $^{63}$ 
A

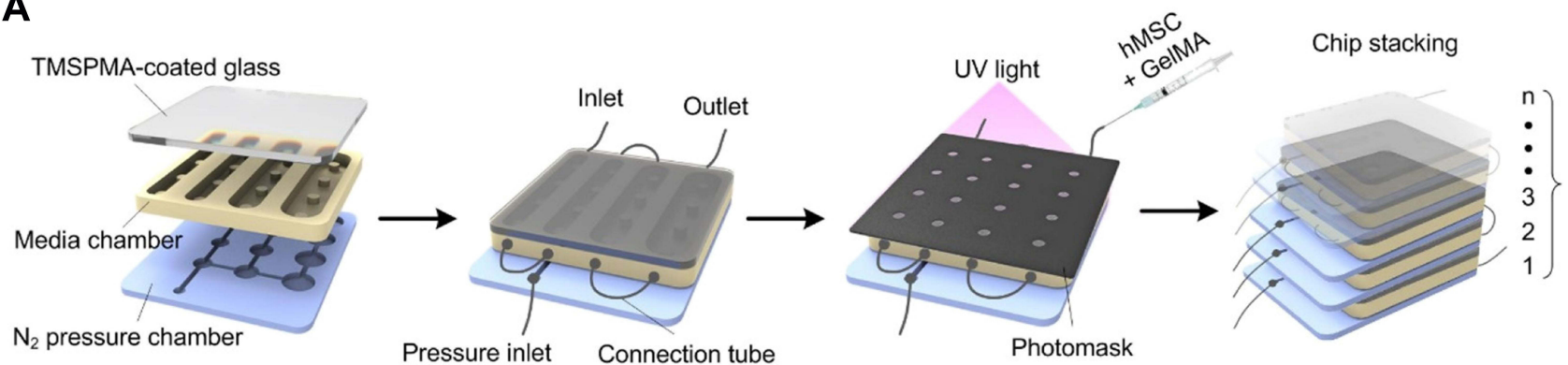

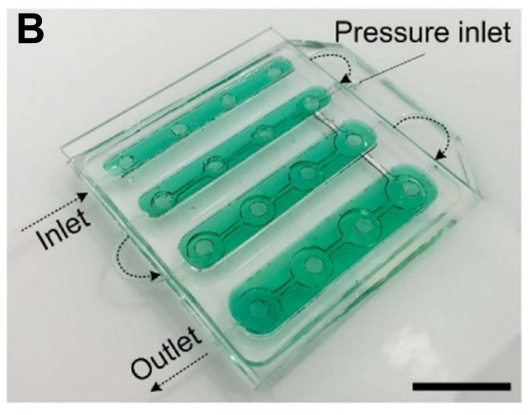

$\mathbf{E}$

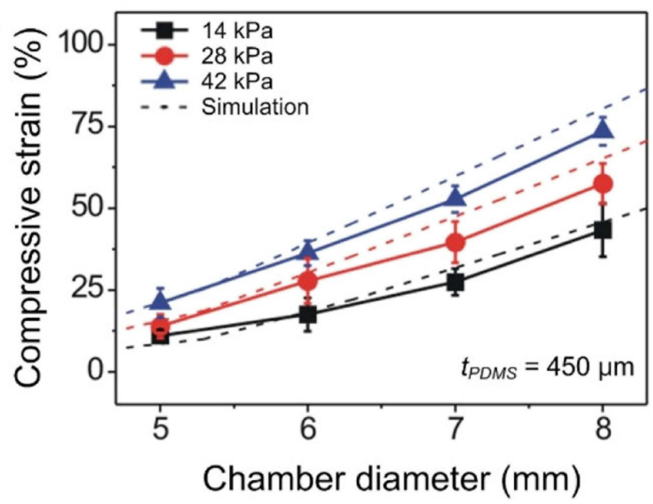

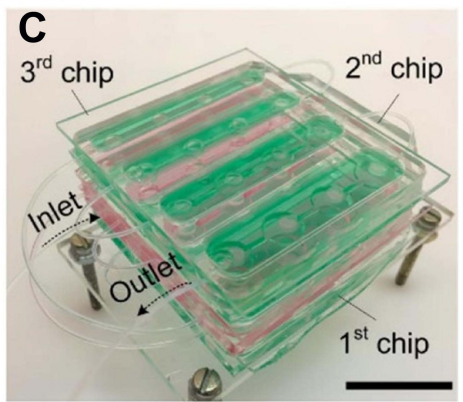
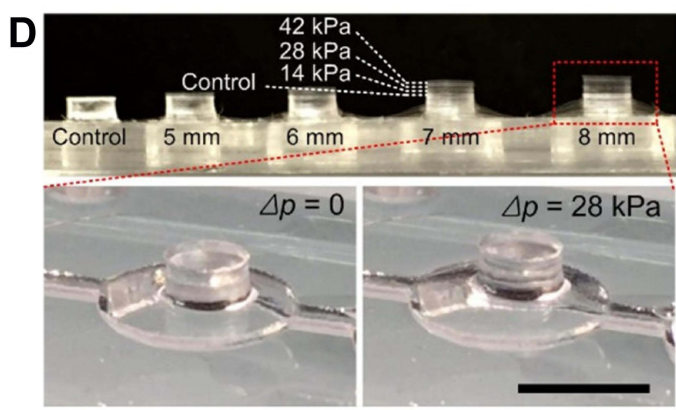

F

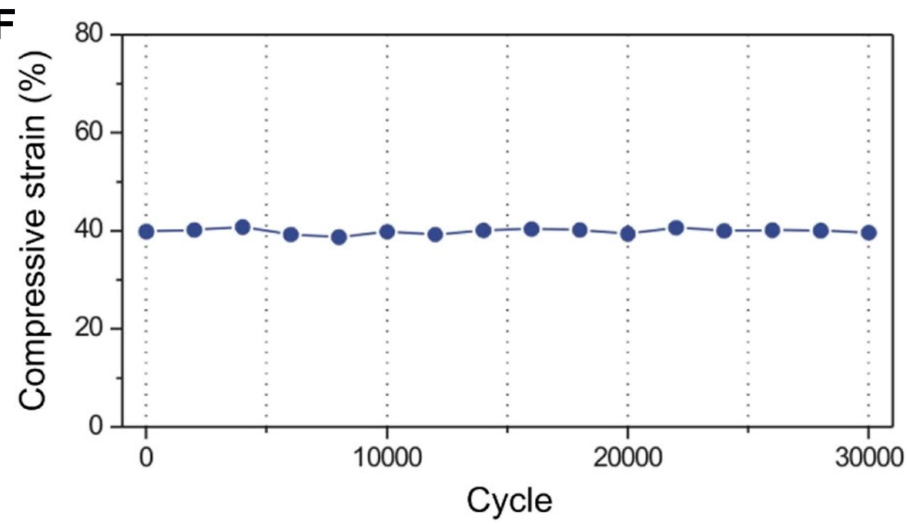

Figure 2 Dynamic compression bioreactors for the combinatorial screening of 3D cellular behaviors. (A) Schematic illustration of the interconnectable bioreactor fabrication process. Photographs of a (B) single and (C) interconnected bioreactor. Scale bar $=2 \mathrm{~cm}$. (D) Vertical displacement of posts depends on the pressure chamber diameter and amount of applied N2 pressure. Scale bar $=5 \mathrm{~mm}$. (E) Experimental and simulation data of compressive strains with $450 \mu \mathrm{m}$ PDMS membrane with different pressure chamber diameters and applied N2 pressures. (F) Stability test of the dynamic compression bioreactor over 30,000 cycles of compression with 14 kPa N2 pressure and an $8 \mathrm{~mm}$ pressure chamber diameter. Reproduced with permission from Seo J, Shin J-Y, Leijten J, et al. Interconnectable dynamic compressionbioreactors for combinatorial screening of cell mechanobiologyin three dimensions. ACS Appl Mater Interfaces. 2018;10(16):13293-13303.63 Copyright @ 2018 American Chemical Society.

\section{Materials}

\section{Natural Sources}

In general, polymers derived from animals or microorganisms are widely used in developing in vitro models due to their excellent biocompatibility (natural enzymesmetabolized degradation) and complex biologically active sites. $^{44,45}$ Several classic examples of these natural polymers include hyaluronic acid (HA), collagen, and alginate, among others. HA is a glycosaminoglycan generally regarded as an inflammation regulator for tissue repair. $\mathrm{HA}$ is increasingly recognized as a promising material for fabricating articulation models due to its good biocompatibility and anti-inflammatory properties. In addition, HA plays a crucial role in forming dendritic cells, $\mathrm{T}$ cell proliferation, and the resultant immune synapses contributed to adaptive immunity and homeostasis of the tissues. ${ }^{46,47}$ In addition to generating 3D models, the injection of HA can lubricate and nourish articulation to effectively inhibit arthritis pain, enhance bone density, repair, and replenish cartilage tissues. To this end, chitosan is a partial deacetyl-based derivative of chitin, which is the main component of crustaceans. Due to the low antigen resistance, the natural polysaccharide chitosan and chondroitin sulfate are employed to generate 3D porous 
models. Further, these models are modified with an HA coating to enhance cell response and effectively deliver bone morphogenetic protein (BMP)-2. ${ }^{48}$ Chitosan-based materials have been proven to be ideal bioactive materials because of their outstanding properties, such as the ability to form different structures and the processability that can be modified with various bioactive materials, as well as their excellent biocompatibility and biodegradability. ${ }^{49,50}$ So chitosan has been widely applied in generating these articulation-based models, such as blood anticoagulant, ${ }^{51}$ articulation nerves, ${ }^{52-54}$ articulation bone. ${ }^{55}$

Collagen is widely applied for engineering blood vessels,${ }^{53}$ bones, ${ }^{54}$ cartilage,${ }^{56}$ tendons, ${ }^{57,58}$ ligaments, ${ }^{59}$ and skin. ${ }^{60,61}$ The hMSCs encapsulated collagen hydrogel was prepared to promote protein-induced collagen fiber mineralization. ${ }^{62}$ By adding soluble $\mathrm{Ca}^{2+}, \mathrm{PO}_{4}{ }^{3-}$, and nucleic inhibitor-bone bridge protein, the hydrogel simulated the inner and outer nano-mineralization profiles of natural bone, indicating that the fabricated microenvironment with natural polymer could substantially stimulate bone differentiation. Bersini and coworkers prepared collagen hydrogels containing bone cells on microflow control devices. ${ }^{63}$ Compared with cell-free collagen hydrogels, the CXCL5/CXCR2 system could effectively invade human mammary MDA-MB-231 cancer cells. ${ }^{64}$ Alginate, a natural dialdehyde acid, is often extracted from the cell wall of brown seaweed or kelp. ${ }^{59}$ Galuzzi et al developed in vitro alginate-based 3D OA model, demonstrating that the nasal cartilage cells encased in alginate beads could produce increased levels of glycosaminoglycans (GAGs). ${ }^{65}$

\section{Decellularized Matrix}

In addition to the extracted natural polymers, the decellularized extracellular matrix (dECM) of tissues and organs can support the microenvironment required by multiple cells in articulation and provide an environment for multicell synergy. Compared with other tissues, the fabrication of articulation units has requirements on sufficient mechanical properties (hardness and yield stress) and specific physicochemical properties of the materials. ${ }^{66,67}$ However, the application of tissue engineering still has certain limitations, such as uncontrollable degradation and insufficient mechanical properties. The current methods for preparing dECM include various physical and chemical methods. In the physical methods, the applied mechanical force may destroy the inherent structure of bone and cartilage acellular structures during the fabrication. Therefore, the fidelity of the shape and the affinity to the cell are usually complementary to each other, becoming a significant challenge for the model design. The use of surfactants in the chemical methods may easily bind to some proteins, in which improper usage and in large residual doses may affect the cell growth. In recent years, there have been a variety of chemical modifications to overcome this difficulty. From this point-of-view, the articulation models made of $\mathrm{dECM}$ provided a perfect microenvironment for multiple cells. ${ }^{68,69}$ An essential difference between $\mathrm{dECM}$ and other materials is its diversity in offering distinctive spatial distribution of functional and structural constituents. The dECM can be vividly called the physiological reservoir of various biomolecules, regulating the adhesion, proliferation, migration, and differentiation of cells. ${ }^{70}$ In general, the ECM comprises two types of secretory macromolecules, fibrin, and glycoproteins, such as proteoglycan, fibronectin, and laminin. ${ }^{71,72}$ These cell-related proteins play an essential role in providing strength and space-filling functions, regulating protein complexes, participating in cell signal transduction, binding growth factors, and promoting cell adhesion. dECM synthesizes natural tissues, which can retain the nutrients required by cells to the greatest extent. ${ }^{73}$ Decellular substrates take automatic object extracellular substrates. The unique mesh-like structure of ECM provides a primary microenvironment for cell growth and development through cell proliferation, differentiation, migration, and intercellular communication. Notably, the dECM prepared after extraction offers no signs of immunogenicity ${ }^{74}$ and can be applied among different animals to introduce multicellular substrates into hydrogels to significantly improve the models' biosafety. ${ }^{75}$ Notably, the deposited cells in dECM resulted in an improved survival rate. They possess a more robust ability to induce the cartilage differentiation of BMSCs fibers and produce a fiber with dECM bio-ink over other treatment groups. ${ }^{76}$ An ECM derived from acellular periosteum tissue was prepared by the VoytikHarbin method and evaluated its immunomodulatory effect at different stages in bone repair, discovering the recruitment of BMSCs by dECM. ${ }^{77}$

\section{Artificial Sources}

An ideal material for joint unit tissue engineering should possess the following features of definite biocompatibility with minimal toxic responses, promoting biological activity, excellent mechanical and degradation properties to 
avoid stress shielding phenomenon, and interconnected porous structure conducive to cell proliferation, blood vessel formation, and nerve innervation. With the advancement of materials science, diverse materials and their composites have been developed for bone tissue engineering and related applications. In general, the most commonly employed biomaterials include bioceramics and polymers and their composites. ${ }^{78-80}$ Although the materials from natural sources are safe, they suffer from certain limitations in terms of immunogenicity, homogeneity, and availability. Several efforts have been dedicated to synthesizing various materials mimicking them concerning the structure and function to solve these problems. Numerous materials from synthetic sources offer the advantages of controllable properties, high stiffness, and augmented performance. For example, synthetic hydrogels possess similar functionalities as biologically derived gels. Synthetic materials for 3D modeling have attracted much attention due to their better mechanical properties, strong plasticity, controllable degradation rate, and diversified production. Nevertheless, the corresponding disadvantages include relatively poor biocompatibility, reduced cell affinity, and the toxicity of the degradation products of the $3 \mathrm{D}$ model to a considerable extent.

Considering these aspects and facts, it is imperative to choose the suitable synthetic material as the articulation has a certain degree of hardness compared with other tissues. Poly(lactic-co-glycolic acid) (PLGA) is often generated by the random polymerization of two monomers, lactic acid, and glycolic acid, resulting in the highly functional degradable organic compound. Owing to excellent biocompatibility, cyst-forming, and membrane-forming properties, PLGA offers excellent prospects in the articulation 3D articulation model. However, PLGA with crystallinity, weak hydrophilicity, and low water absorption often results in a slow degradation rate and is not conducive to the colonization and growth of cells, requiring some surface modification. Water-in-oil-in-water $(\mathrm{W} / \mathrm{O} /$ W) emulsion-assisted gelatin-based PLGA microspheres are often used for creating $3 \mathrm{D}$ models by the double emulsion method. ${ }^{81}$ Notably, the average particle size and the pore diameter of the designed PLGA microspheres are often determined by the injection rate and the volume of the gelatin concentration. The resultant PLGA microspheres possess spherical-shaped structures with porous architectures and interconnecting windows, promoting cell penetration and nutrient exchange. ${ }^{82}$ Polycaprolactone (PCL) is a white opaque solid with certain rigidity, and strength, as well as typical resin characteristics. PCL has good compatibility with polymer materials and adapts to the stiffness of cartilage that can be used as a modifier to improve some other polymers. The bioprinted PCL scaffold simulates the circumferential and radial collagen orientation in the natural meniscus. This cell-free model shows excellent biocompatibility and produces good biomechanical capabilities very close to the natural meniscus. $\beta$-calcium phosphate modified PCL promotes alkaline phosphatase activity and mineralization determination for bone defects.

Previous studies indicated that the synthetic PEG dimethacrylate hydrogel could be used in a mechanical loading system. The external mechanical stimulation was an effective modulator of the differentiation of hMSCs to chondrocyte phenotype. ${ }^{83}$ The system included multiple cell types in the development of OA. However, it is significant to study the interactions between cells at the molecular level during the development of OA. In addition, a large amount of water in the hydrogel may impact the structural integrity of the gel anchoring the substrate. The appearance of biological hydrogel supports the proliferation of chondrocytes and the production of ECM and contributes to the growth and mineralization of osteoblasts, providing a potential model for studying the activity of the cartilage interface. ${ }^{84,85}$ To this end, polyvinyl alcohol (PVA) with good biocompatibility has been used in fabricating articulation units owing to its equivalence in terms of the viscoelasticity of articular cartilage and meniscus. $^{78}$ However, the applicability is often nonconducive to cell colonization and growth because of its weak protein adsorption capacity. In a case, the LTi-DAPVA composite hydrogel with the hydrophilic surface was compounded with Ti6Al4V alloy, resulting in excellent dual-phase lubrication and synergistic effects. The friction coefficient of the LTi-DA-PVA sample could be as low as 0.01 , which was as low as the friction of natural cartilage. Similar coefficients provided an effective method for the surface wettability and tribological properties of artificial articulation. $^{86}$

Hydrogel is a common synthetic hydrated material based on different polymers, mimicking the ECM-like environment in terms of controllability, biodegradability, porosity, biosafety, and low immunogenicity. ${ }^{87}$ These hydrogel-based 3D models provide the ability to alter biochemistry, ${ }^{88}$ matrix elasticity, microstructure pore size, and pore interconnectivity, affecting cell vitality and its ability to secrete ECM. ${ }^{84}$ Similar to other polymeric 
constructs, these synthetic hydrogels have enhanced key cartilage markers, promoting cartilage formation, such as type I collagen and non-collagen. ${ }^{89-91}$ Moreover, the synthetic hydrogel can be fine-tuned by various chemical modifications that promote cartilage production through augmenting cartilage transforming growth factor- $\beta 3$ (TGF- $\beta 3$ ) and the expression of type II collagen and GAGs. ${ }^{54,92}$ Undoubtedly, the choice of scaffolding material leads to a new path in developing the $3 \mathrm{D}$ articulation models. Hydrogel derived from alginate, hyaluronic acid, gelatin, chitin can promote cartilage activity and accelerate value-added while enhancing the expression of type-I collagen, aggregate protein polysaccharides, and Sox $9 .{ }^{93-96}$ In several instances, such hydrogels have been manufactured using bioprinting to improve feasibility and reducing the need for multi-step manufacturing processes and crosslinking with potentially toxic constituents. ${ }^{97}$ Gelatin methacrylate (GelMA) is photosensitive hydrogel material, in which the visible light stimulates the curing reaction to form a 3D structure with a certain strength for the growth and differentiation of the incorporated cells. In a case, Shi et al created a new type of ultraviolet radiationassisted osteogenic peptide hydrogel based on GelMA and osteogenic growth peptide (OGP). ${ }^{98}$ GelMA could form a light atomized OGP hydrogel with good photomechanical properties and promote bone regeneration. In another case, Weitz and coworkers used microfluidics to prepare photo-crosslinked microspheres, which provided a suitable microenvironment for the growth of bone marrow stromal cells to achieve the regeneration of injectable bone tissue. ${ }^{99}$ In many other instances, GelMA-based bioink was developed as a personalized treatment strategy for bone regeneration. ${ }^{100}$

\section{Microengineering Strategies Microfluidics}

The microfluidic technology has garnered enormous interest from researchers in fabricating various 3D architectures by precisely controlling the fluids at the microscale, for instance, polymer microspheres with good dispersion, as well as controlled particle size, and shapes that could be applied for encapsulating the biological entities such as cells. ${ }^{101}$ Recently, several efforts have been dedicated to utilizing single-phase and bipolar methods to prepare PMs. In addition to being simpler and more feasible, the singlephase method offers another advantage of avoiding the damage to cells caused by later bipolar multiphase residues. The two-phase method creates microspheres with 3D and highly complex internal porous structures, simulating the physiological microenvironment. By adjusting various processing (flow rate and ultrasonic power) and formulation parameters (porogen and initial precursor concentrations), the diameter of the microsphere and the size of the pores can be adjusted while fabricating microspheres.

The microfluidics-based culturing approaches typically generate the micro-sized platforms that reflect the physiology of articulation structures, such as simulating the articulation bone, the cartilage part, and the sliding membrane, along with the characteristics of spatial stride and mechanical load. ${ }^{43,102}$ The stringent control over the fluidic flows offers inherent flexibility in the architectural design, allowing the associated concentration gradient and cell space structure to be controlled. ${ }^{103}$ In this vein, the microfluidic technology can generate various 3D designs, such as spherical, tubular, and chip models, which provide infinite possibilities for bionic complex and diverse articulation unit models.

Depending on the porosity and morphology attributes, these microspheres generated from the microfluidic technology have been applied in different biomedical applications. For instance, microspheres with small diameters and small apertures are primarily used as drug carriers for sustained release effects. In contrast, the large-sized PMs as in vitro models are mainly used to load large biomolecules (for instance, proteins) and cells for minimally invasive tissue engineering and drug screening. ${ }^{104,105}$ In this case, a microbot system with an average diameter of 150-200 $\mu \mathrm{m}$ was designed for encapsulating human fat MSCs and efficiently delivering them using magnetic-driven technology. Different polymerization methods were employed to produce hydrogel particles with different uniformities towards encapsulating cells (Figure 3A and B). It could be observed that different polymerization methods resulted in the difference in the uniformity and size distribution of hydrogel particles (Figure 3C and D). However, the SEM observations of the particles prepared in different oil phases using Novec 7500 showed uniform-sized particles (Figure 3E). ${ }^{99}$ In recent times, the microspheres cultured with cartilage cells as scaffolding systems have begun to be widely used. For instance, the gelbased 3D microspheres as cell models are expected to be applied for exploring the mechanistic elucidations of drug penetration and performance efficacy. The prepared gel microsphere models can be transferred to 96 or 384 well plates, providing the possibility for high-throughput drug screening. 
A

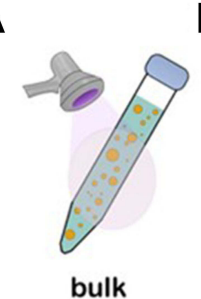

bulk
B

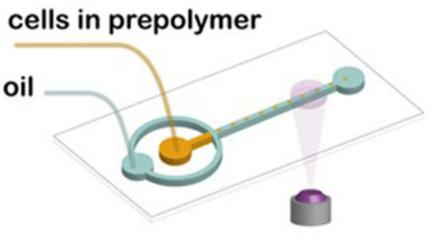

in device
D

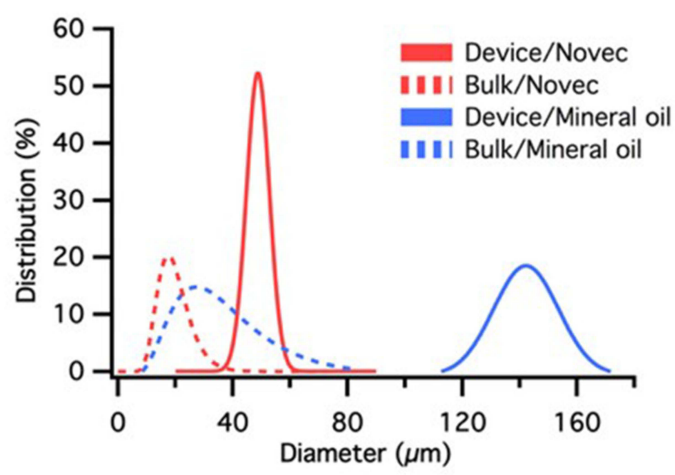

C

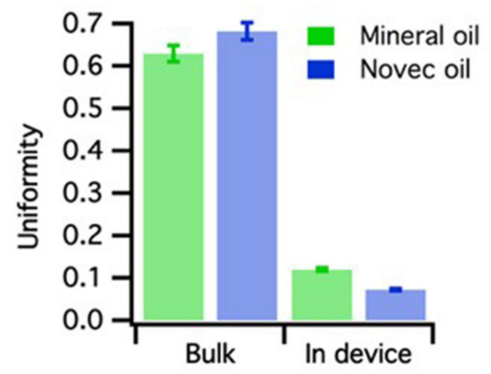

E

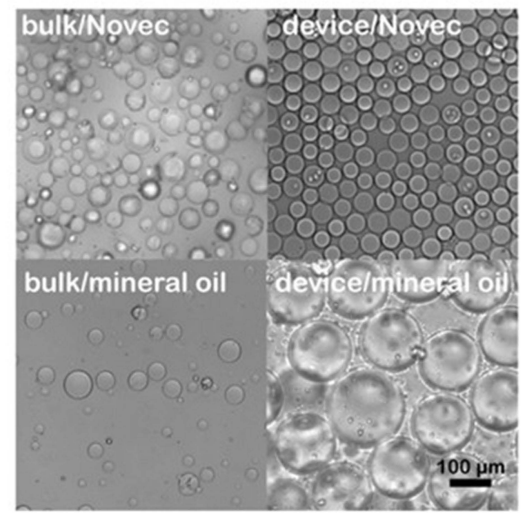

Figure 3 Cell encapsulation in bulk (A) and in a cross-flow microfluidic channel (B). Uniformity (C) and size distribution (D) of hydrogel microparticles produced using different polymerization methods. (E) Images of microparticles obtained through different methods and oil phases: in bulk and in device using Novec 7500 and mineral oil. Reproduced with permission from Xia B, Krutkramelis K, Oakey J. Oxygen-purged microfluidicdevice to enhance cell viability in photopolymerized PEG hydrogelmicroparticles. Biomacromolecules. 2016;17(7):2459-2465. ${ }^{99}$ Copyright (C) 2016 American Chemical Society.

Microspheres controlled by optical cross-linking microfluidics provide an excellent microenvironment for the growth of bone marrow matrix stem cells to achieve injectable bone tissue regeneration and drug screening.

Interestingly, the concept of microfluidics can also be used to create tubular porous structures, to mainly construct biomimetic blood vessels. At the same time, for the design of microflux control channels, changes in the size of microchannels in the equipment, geometry, and solution viscosity can be further applied to prepare bipolar blood vessels and other multi-stage structures. In a case, Jusoh et al proposed a network of 3D microvascular vessels in the ECM with hydroxyapatite (HA) to design and manipulate vascular bone tissue models in microflow control devices. In detail, the gelatin modified seaweed acid was applied as an injectable short rod stent structure evenly wrapped with human umbilical vein endothelial cells (HUVECs). These biocompatible hollow structures with HUVECs were fabricated using the citric acid as a crosslinker for seaweed gel, in varying degrees of fixation, thus successfully inducing HUVECs within the scaffold to migrate outwards forming a tube cell layer to simulate articulation vascular system. ${ }^{106}$ The osteoblasts are cultured together with vascular microorganisms to form an articulation unit, adding inflammatory factors for high fluoride screening of the drug to verify the effectiveness and safety.

The concept and principles of the microfluidic technology have been employed to assist in organ-on-chip perfusion approach. A complex 3D channel simulates a more complex physiological micro-environment, and the control over the micro-flow accurately monitors the performance, ensuring the normal operation of the whole system. With the assistance of digital control, the microfluidic chips can be used for high-throughput drug screening and bioseparation. In an instance, Gao et al designed a dynamic, low-key discharge-type tibia femur model by integrating microflow control technology. ${ }^{107}$ The concentration gradient between tissue was the key to cell metabolism, and the ordinary model could not cope with complex physiological changes. The use of microfluidic chips to culture horse chondrocytes established a physiological nutrient diffusion gradient simulation mechanism and quickly and efficiently formed knee cartilage tissue.

\section{Bioprinting}

In recent times, bioprinting has garnered enormous attentiveness from researchers owing to its ability to conveniently manufacture various types of versatile multi-tissue 
constructs with high accuracy and repeatability. ${ }^{108-110}$ Combined with computer-assisted modeling, bioprinting can be employed to generate complex (multi-layer) articulation parts in multiple dimensions with great potential for joint repair (cartilage defects), inducing bone regeneration, and drug screening for exploiting the pathological conditions. $^{32,111}$ Notably, several hierarchical articulation models have been fabricated using bioprinting include multi-functional layered scaffolds, tubular stents. ${ }^{112}$ For example, three layers were created using near-field direct writing techniques: surface cartilage (S), deep cartilage (D), and lower sternum (B), in which the PCEC stratified 3D modeling (Figure 4A and B), filled with interstitial stem cell GelMA hydrogels and region-specific growth factors (Figure 4C). ${ }^{113}$

By applying the composite 3D modeling, bioprinting can regenerate both cartilage and bone composite in vitro, proving that the bionic structures that mimic natural osteoporosis tissue can enhance the regenerative feasibility of osteoporosis tissue. In a case, Ozbolat et al applied composite bioprinting using cartilage cells different from human fat stem cells to prepare tissue beams as bioprinted inks. Moreover, the authors constructed the regional stratification of artificial cartilage to improve the mechanical properties and anatomical structure, providing a closedloop strategy for cartilage repair. ${ }^{114}$ Using bioprinting technology, a series of optimized bracket manufacturing strategies were studied to precisely control the structure, degradation rate, and mechanics, providing a new type of natural biodegradable bracket regeneration. ${ }^{115}$ Cartilagelike tissue was printed with the cell microspheres bioink, which contributed to the further clinical development of cartilage replacement and regeneration. ${ }^{116,117}$

In this vein, coaxial bioprinting can be applied to fabricating bionic tubular structures. However, the applicability of this coaxial-based printing is challenging in achieving the long-term culture due to the lack of blood vessels in the printed articulation bone tissue. ${ }^{118-122}$

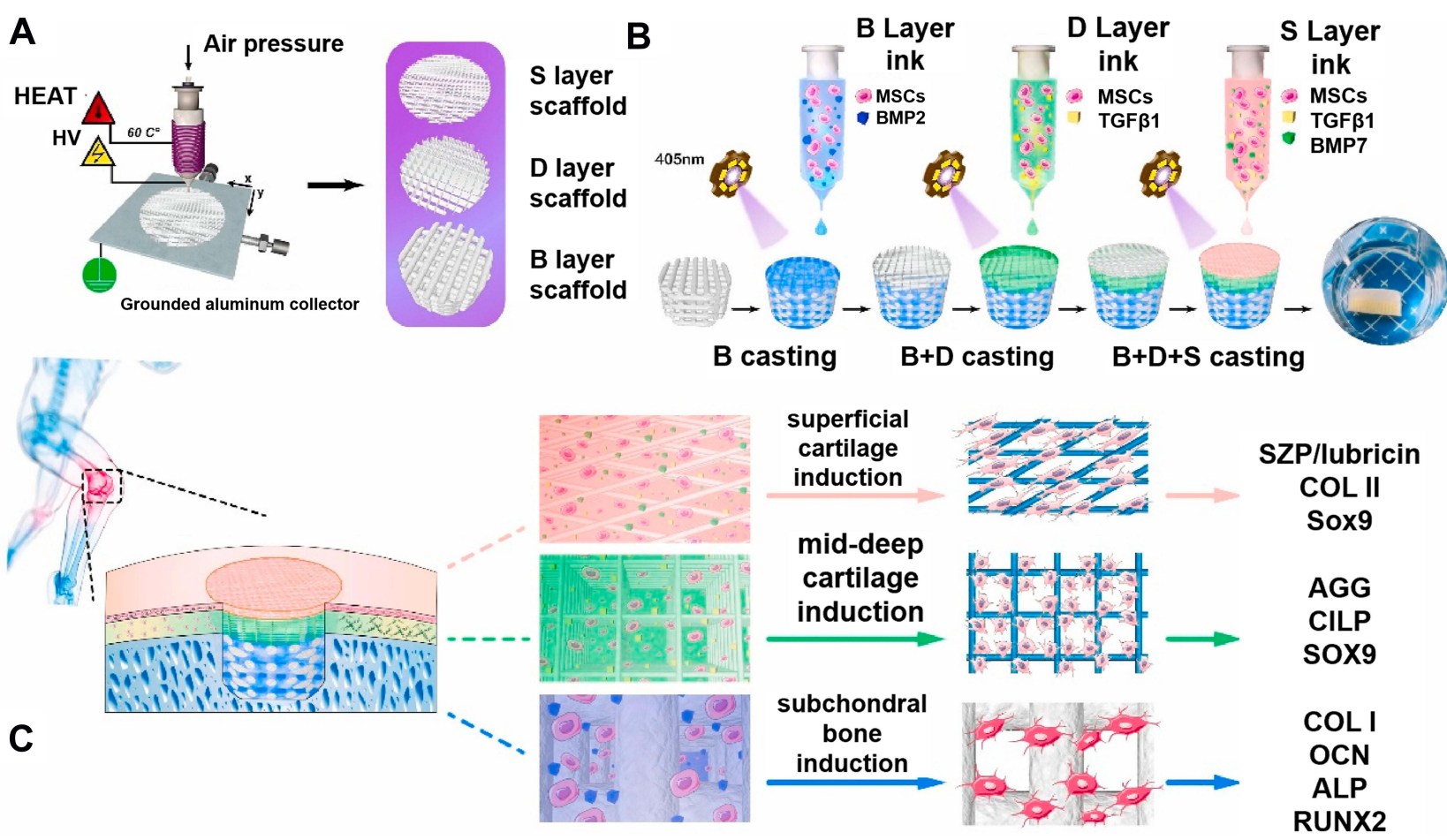

Figure 4 Schematic diagram showing the preparation process and application scenario of the tri-layered fiber-reinforced and GF-loaded hydrogel constructs: (A) Fiber networks for the S, D, and B layers were fabricated by melt-electrowriting (the high voltage was removed when fabricating the B layer 3D model); (B) The integrated trilayered composite was constructed using the UV-assisted, stepwise infiltration and crosslinking procedures. As shown, with the assistance of UV-crosslinking, the cell and growth factor-laden GelMA hydrogel precursor solution of the respective layer was successively infused into the fiber networks for the B, D, and S layers to construct the tri-layered fiber-reinforced hydrogel composite; (C) Schematic illustration for application of the tri-layered composite 3D model in layer-specific osteochondral tissue induction and regeneration. The combined effect of layer-specific fiber arrangement and GF delivery enabled the layer-specific induction of BMSCs to exhibit zonal cell phenotypes and ECM compositions resembling those of native osteochondral tissue. Additionally, the inclusion of the $S$ layer could impart the regenerated cartilage with a more lubricating and wear-resistant surface, which is a 3D model for functional osteochondral regeneration. Reproduced with permission from Qiao Z, Lian M, Han Y, et al. Bioinspired stratified electrowrittenfiber-reinforced hydrogel constructs with layer-specific inductioncapacity for functional osteochondral regeneration. Biomaterials. 15402021;266(6338):120385. ${ }^{113}$ (C) Copyright 2020, Elsevier. 
Scientists continue to develop new biomaterials and printing techniques to build networks of blood vessels within bioprinting structures. ${ }^{112}$ However, the vascular networks produced by other traditional and indirect methods are often larger than $100 \mu \mathrm{m}$ in diameter, making it challenging to reconstruct fine capillary networks. Moreover, the post-printing process, including the removal of sacrificial materials and hollow endothelial networks, is complex and time-consuming. In contrast, direct bioprinting of tubular networks can be more promising and an effective alternative over the other indirect methods. In this regard, due to biocompatibility, several natural biopolymers have been applied in vascular bioprinting, such as fibrin, collagen, gelatin, and cell-matrix. However, the complex matrix properties of protein-derived biological materials complicate the fine regulation of their vascular characteristics. In addition, the composition of these materials is often affected by batch changes. These challenges have guided the possibilities and feasibility for further developing in vitro articulation unit models. Integrating hydrogels, ceramics, and polymer materials to mimic calcium phosphate $(\mathrm{CaP})$ in the mineral stage of skeletal muscles by near-field direct writing and extrusion printing technology has resulted in innovative hierarchical composites. In this case, the near-field direct writing was applied to prepare polymer meshes, repair in ceramic inks, and embed GelMA tubular loading cells as capillaries. ${ }^{123}$ Based on DLP printing technology, several researchers have successfully prepared a bionic bone 3D model with a complete layer of bone structure and induced osteoporosis, angiogenesis, and neurogenic differentiation of in vitro analog articulation tissue. The bionic bone model with complete stratified haver's bone structure has been successfully prepared. By changing the parameters of haver's bone-like structure, better control of the pressure strength and porosity of the bracket was achieved, providing gaps for new blood vessels and nerve growth and differentiation and promoting blood vessel growth and new bone formation in the body. ${ }^{117}$

\section{Electrospinning}

Electrospinning is often applied for manufacturing fiber supports in the range of micron-sized scaffolds to nanosized networks. In general, the electrospinning equipment is facile, consisting of three parts: a high-voltage power supply unit, a syringe/coaxial with a metal needle, and a ground metal collector. ${ }^{124}$ The morphology of the resultant fibers can be adjusted or simulated by controlling various processing parameters, such as solution conditions, distance between tip and plate, electric field strength, nozzle size, and methods for collecting sample plate and rotating Mandrel and collection speed. ${ }^{125,126}$ Typically, the electrospraying-based 3D modeling results in random porous nanofibrous membranes. Electrostatic spinning possesses broad prospects in applying membrane and microtubule structure and is mainly used in the joint capsule, half-moon plate, vascular nerve, and other models. The nanofibrous material produced by electrospinning technology mimics the microstructure of the 3D modeling outside the bone cells due to its natural porous structure. ${ }^{127}$ This section summarizes the typical applications of electrostatic spinning in fabricating the articulation unit models of fiber membranes and tubular brackets.

As mentioned earlier, electrospinning is often utilized as a small-scale manufacturing method of 3D fibrous materials that mimic the structural characteristics of natural ECM and deliver cell and other bioactive molecules. ${ }^{128}$ By combining electrostatic spinning with self-assembly technology, a composite structure of bionic bone membrane was constructed for slowly releasing vascular endothelial growth factors. The SEM observations indicated that the resultant PLGA fibers were thinner and formed more nodes. The periosteum obtained could repair articulation bone tissue unit by this method. Chen et al prepared a 3D model based on SF/CS/nHAP loaded with BMP-2 by the electrospinning, which could be a promising framework for bone tissue engineering (Figure 5). ${ }^{129}$ The bionic meniscus prepared by the wet electrospinning method was used to construct a suitable pore size and sufficient mechanical support through a cellfree 3D electrospinning using $\mathrm{PCL} / \mathrm{silk}$ fibroin $/ \mathrm{Sr}^{2+} 3 \mathrm{D}$ model, protecting cartilage and delaying the development of OA. Several reports demonstrated the modification of the surface of PCL nanofibers with gelatin, then tested their biocompatibility with nanotube cells, indicating that nanofibrous membranes replaced with gelatin significantly promoted the migration and proliferation of embryonic cells towards vascular tissue engineering. ${ }^{130}$ To produce vascular models, the resultant fibrous materials by the electrospinning technology can be loaded with endothelial cells. In this vein, several reports demonstrated the fabrication of a composite bionic bone mold that could release VEGF slowly, providing a new way of thinking for articulation simulation. ${ }^{131}$ These vascular 3D models could be cellularized for angiogenesis or mature human body mixtures. 


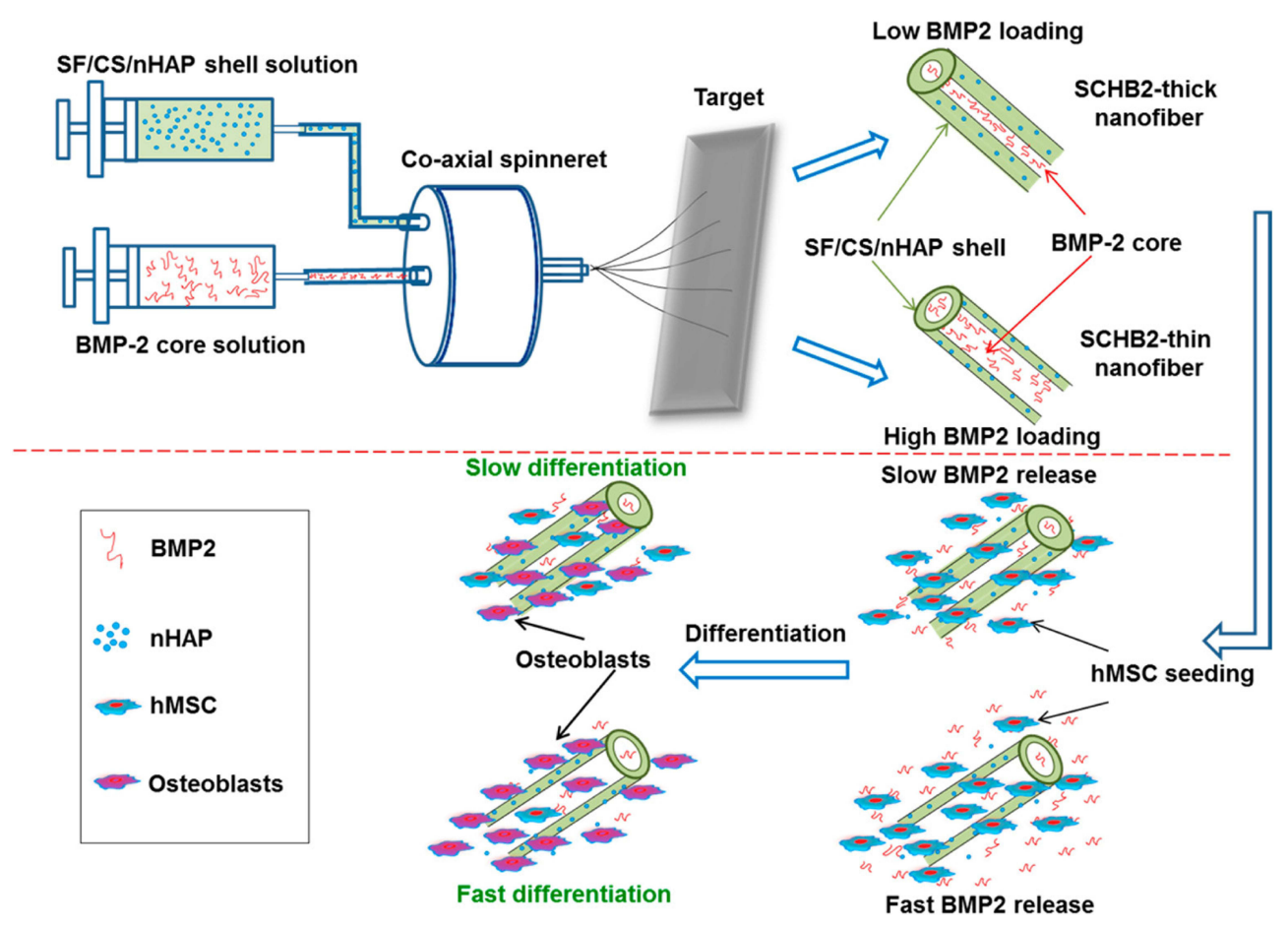

Figure 5 Schematic Representation of the Preparation of SCHB2-Thick and SCHB2-Thin NFMs through Coaxial Electrospinning and Their Influence on hMSCs. Reproduced with permission from Shalumon KT, Lai GJ, Chen CH, et al. Modulation of Bone-specific tissue regeneration by incorporating bone morphogeneticprotein and controlling the shell thickness of silk fibroin/chitosan/nanohydroxyapatite core-shell nanofibrous membranes. ACSAppl Mater Interfaces. 20I5;7(38):21 I70. ${ }^{129}$ Copyright $\odot 2015$ American Chemical Society.

\section{Biomineralization}

In general, the 3D models for bone tissue engineering are fabricated by simply imitating the chemical composition of natural bone and recreating the hierarchical structure of natural bone tissues. The biomimetic mineralization approach is one of the biomimetic fabrication strategies committed to synthesizing the natural bone-like substitute on the nano level to obtain articulation models. ${ }^{132,133}$ These substitutes with good biocompatibility and bioactivity provide host cells with a natural microenvironment, thereby inducing endogenous bone regeneration. ${ }^{134,135}$ In a case, Bertassoni et al developed a collagen hydrogel encapsulated with hMSCs, promoting protein induction by adding soluble $\mathrm{Ca}^{2+}, \mathrm{PO}_{4}{ }^{3-}$ and nucleation inhibitors bone bridge protein collagen fiber to simulate the osteogenesis and cleavage of natural articulation bone. The authors demonstrated that the microenvironment was sufficient to stimulate bone differentiation of hMSCs and form hMSCs-supported capillaries in the body. ${ }^{63}$ In another case, a new material system was discovered by the natural mineralization process in coral reefs and studied the biomineralization properties of cartilage composite photocurable bioinks for a gel 3D model. In this system, the piezoelectric bracket was stimulated by external mechanical loads to produce a proportional charge and using these charges as a signal to induce mineral ions in the surrounding medium to mineralize and deposit on the 3D model, thereby enhancing the mechanical properties of the load part for self-hardening. ${ }^{65}$

\section{Biomedical Applications Drug Screening}

Over the years, similar to other drug evaluation strategies in the preclinical stage, researchers have adopted the 2D models to study the therapeutic efficacy of various drugs for articulation diseases. In this regard, the 2D monolayer cell culture models played essential roles in developmental biology, histopathology, disease mechanisms, drug discovery, mass protein production, and tissue engineering, as well as regenerative medicine. However, these 2D culture models suffer from several limitations, such as failing to mimic the complex in vivo environment in terms of anatomical and physiological characteristics, resulting in the differences while extrapolating their experimental results to in vivo. To this end, the in vitro 3D models have presented great potential as a preclinical testing platform for drug development and addressing the limitations of 2D monolayer testing tools. ${ }^{136-139}$ These 3D models of dense 
cell masses with reproducible sizes, morphology, and necrotic core formation, are typically prepared through different tissue engineering strategies to simulate natural tumors and show higher invasiveness and drug resistance than ordinary 2D culture. Although the 3D models for drug screening offer apparent advantages over the traditional 2D models and animal models, they still suffer from certain limitations in terms of morphological and functional attributes. For example, the pore size of a 3D sphere must be optimized with interconnecting windows such that the cells can grow through the pores to fabricate the cellladen spheroids for drug screening. In addition, the surface area plays a key factor in cell colonization.

Moreover, the chondrocytes need to be subjected to a certain stress stimulation to maintain their function. However, it should be noted that there exist specific difficulties in achieving controllable stress stimulation in vitro. Furthermore, in vitro drug screening models require a complete vasculature to maintain biological activity for a long time. The compactness of the structure and organization makes it more challenging to construct vascularization in vitro. We summarized the studies explored in fabricating such unit models in recent years. Based on the inherent physiological and drug response differences between osteosarcoma cells and 3D spherical hydrogels, ECM simulated hydrogel models for tumor modeling in vitro and high-throughput assessment of anti-cancer agents have been prepared. ${ }^{140}$ Bioprinting enables the production of highly functional tissue architectures that simulate the physiological and anatomical properties of the native tissues. Notably, the advances in 3D printing technology enable the precise control of the architectural designs encapsulated with cells of articulation units, such as chondrocytes, stromal cells, bone cells, and synovial cells.

Moreover, these approaches integrate growth factors delivery, body fluid circulation, physical stimulation, including external pressure, oxygen levels, and mobility. However, these structures should mimic the specific functionalities of a linear joint: biomechanics, mobility, and multi-cellular interactions. MSC-based exosomes substantially promote cartilage regeneration through multifaceted mechanisms by synthesizing matrix and attenuating apoptosis and modulating immune reactivity. Owing to these considerations, these elements are grouped in spatial and temporal manners and restructured, mimicking joint organs, allowing research on the pathophysiology of rheumatism, the development of biomarkers, and the screening of new drugs. ${ }^{141}$ In a case, a 3D model was prepared to understand the pathological and physiological mechanisms involved in the destruction of cartilage and subchondral bone during rheumatoid RA, simulating cytokine-induced cell and matrix-related changes and leading to cartilage degradation, as well as bone destruction to ultimately provide preclinical drug screening tools. ${ }^{136}$ In vitro toxicological analysis can significantly benefit from the bioprinting approach as the small molecules (drugs) can be assessed with higher efficiency in a drastically shorter time. In several instances, researchers applied the bioprinting approach to producing cartilage structures that could be used for in vitro drug screening using an expandable "tissue chain" bioprinting model.

\section{Tissue Regeneration}

In general, stimulating the tissue regeneration ability is an effective treatment method for joint diseases. Indeed, the human body possesses the repair potential of the bone and its surrounding microenvironment (including inflammatory cells, endothelial cells, and Schwann cells), thereby restoring the homeostatic functional state and further highlighting the crucial role of bones in mammalian physiology. In this vein, several reports have demonstrated that the balance of regulatory activity between osteoblasts forming bone and osteoclasts (main cellular components of bone) in terms of dissolving and absorbing bone is responsible for the repairing ability. Moreover, the research on the role of osteoblasts presented the importance of various morphogenetic components, such as BMP, in the process of bone repair and bone development (osteogenesis).

The most commonly employed biomaterials include bioceramics, polymers, and metal-organic framework (MOF). ${ }^{142}$ Several composite biomaterials with layered structures have emerged as promising materials for bone tissue engineering. In a case, a bionic bone with nitrogen-doped graphenehydroxyapatite (NG-HA) mixture and agarose (AG) matrix was prepared by simple hydrothermal/crosslinking/freezedrying, which offered a potential application value in bone tissue regeneration. ${ }^{143}$ Some findings highlighted the significant prospect of 3D in vitro models for tissue regeneration. However, these structural designs still fail to offer a long-term effect. However, the 3D articulation unit models loaded with cell growth factors are expected to solve the dilemma in tissue repair by avoiding complex trauma surgery through these minimally invasive models. ${ }^{144-146}$ Moreover, the injected myelin-filled stem cell models induce bone marrow interstitial stem cells to accumulate in the wound by playing the function of stem cell multi-directional differentiation to repair cartilage. ${ }^{147}$ In articular cartilage, various growth factors or 
active peptides work synergistically to enhance the healing ability of cartilage damage and prevent the occurrence and development of degenerative arthritis. Inducing the directional differentiation of cartilage requires suitable stimulating factors to stimulate and promote cartilage differentiation. It has been proved that many cytokines could induce or stimulate chondrocyte differentiation. This section mainly introduces the application of the in vitro model of loading multiple growth factors and active peptides for articulation repair.

$\mathrm{BMP}$, a functional protein, belongs to the highly conservative TGF- $\beta$ family, which often stimulates DNA synthesis and subsequent cell replication, therefore stimulating the osteogenic differentiation of mesenchymal cells. The BMP activity provided by BMP-2 is necessary for the maturation and maintenance of the mouse knee articulation and reveals the unique role of BMP-2 in knee biology. ${ }^{148}$ In addition, the repair of the knee articulation requires blood vessels to deliver nutrients. The vascular endothelial growth factor (VEGF) can promote the growth of cancer cells. By combining microsol electrostatic spinning with collagen self-assembly technology, Cui et al developed a composite structure bionic bone membrane that slowly releases vascular endothelial growth factor for tissue repair. $^{128}$ Osteogenic growth peptide (OGP) is a polypeptide consisting of 14 amino acid residues, a promoter of a systematic response to bone marrow damage. OGP stimulates the activity of osteoblasts and promotes the growth of osteoblasts and fibroblasts. In a case, Lee and coworkers designed a nano clay-organic hydrogel bone sealant that integrated multiple physical and chemical elements of bone regeneration into the same system.

The assembly of plant compound modified crustal polysaccharides and silicon-rich inorganic nano clay could be used as biocompatible and bone conduction of ECM simulator. ${ }^{149}$ An asymmetric porous membrane with a stacked structure of blades was prepared. The membrane was immersed in the BMP-2 solution to improve cell differentiation and new bone formation (rat model of cervical disc deformity). It was observed that different $3 \mathrm{D}$ structure models resulted in different expression levels of osteogenic differentiation markers (Figure 6A), and more intuitively, through dyeing (Figure 6B). ${ }^{151}$ In this vein, calcitonin gene-related peptide (CGRP), an active peptide, presents the main physiological effect of promoting osteoblastic function, endorsing the formation of osteoblasts, increasing bone activity, and stimulating the proliferation and differentiation of bone cells of origin. Neugebauer and coworkers modeled on collagen II-induced rat arthritis to study the effects of chronic arthritis pain on the expression of calcitonin-related peptide genes in sensory and motor pathways. In addition, the effects of the glucocorticoid drug budesonide on changes in arthritis-induced CGRP were examined. It was evident that the concentration of articulation fluid is positively correlated with the peptides associated with the calcitonin gene by studying the intraarticulation temperature of arthritis and the level of articulation fluid associated with CGRP. ${ }^{150}$

\section{Conclusions}

In conclusion, we have summarized the generation of articulation unit models for diverse biomedical applications. Initially, we presented the development of different in vitro models, including no scaffold, scaffold-based system, and organ-on-a-chip, by applying various materials, engineering strategies, and growth factors or active peptides. In this vein, different microengineering strategies applied in preparing various models with different structures are emphasized, such as PMs for minimally invasive delivery, fibrous membranous shapes for simulating the regeneration of the periosteum, and meniscus, as well as the tubular structures to solve the complexity of blood vessels and nerves in joint bones.

By simulating the near-physiological microenvironment, these 3D models of articulation units have shown their excellent abilities in tissue regeneration and drug screening applications. However, there still exist some challenges in exploring these models for preclinical and clinical applications. First of all, the physical and mechanical properties of the models must be optimized and precisely controlled on-site. Further, research is needed to explore the development of highly porous and strong biomaterials with controlled biodegradation, rate of new bone formation, and ensured cell adhesion and growth. Furthermore, the difficulty of vascularization in model tissues has always been the bottleneck of these in vitro models. Due to the simple integration of the tubular structure and the branch vessel network, manufacturing a $3 \mathrm{D}$ vessel network is still challenging. In fact, the blood vessels with an extensive diameter range are distributed throughout the body. Therefore, a 3D structure with structural complexity with vascularized tissue constructs is still required. Importantly, coaxial bioprinting is challenging to achieve the accuracy of capillary printing, while electrospinning is difficult to control to form a continuous blood vessel structure. It is worth mentioning that one way to promote blood vessel formation is to generate a porous network with interconnecting windows in the scaffold. However, by binding and releasing biologically active molecules with controlled kinetics, vascularization can 


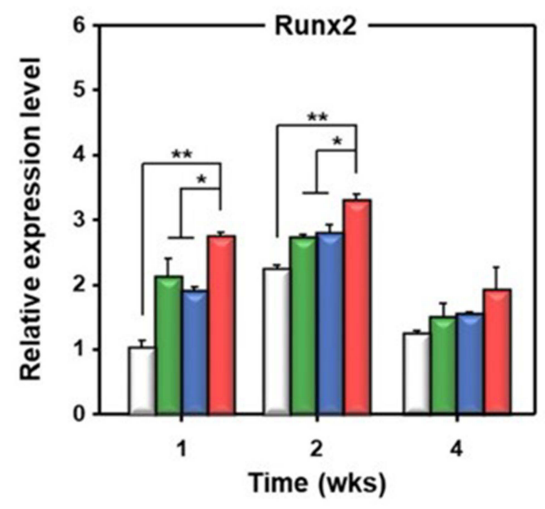

A
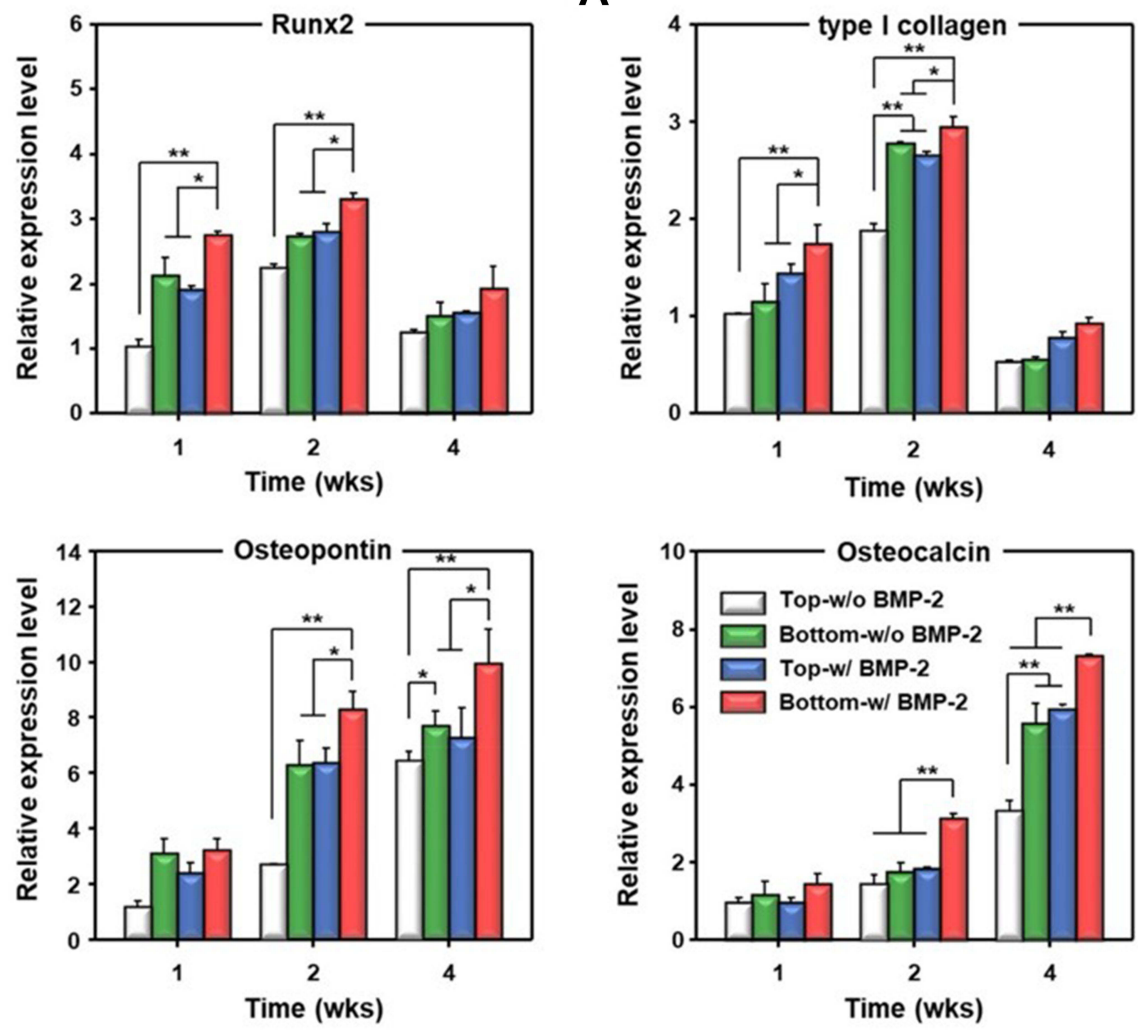

B
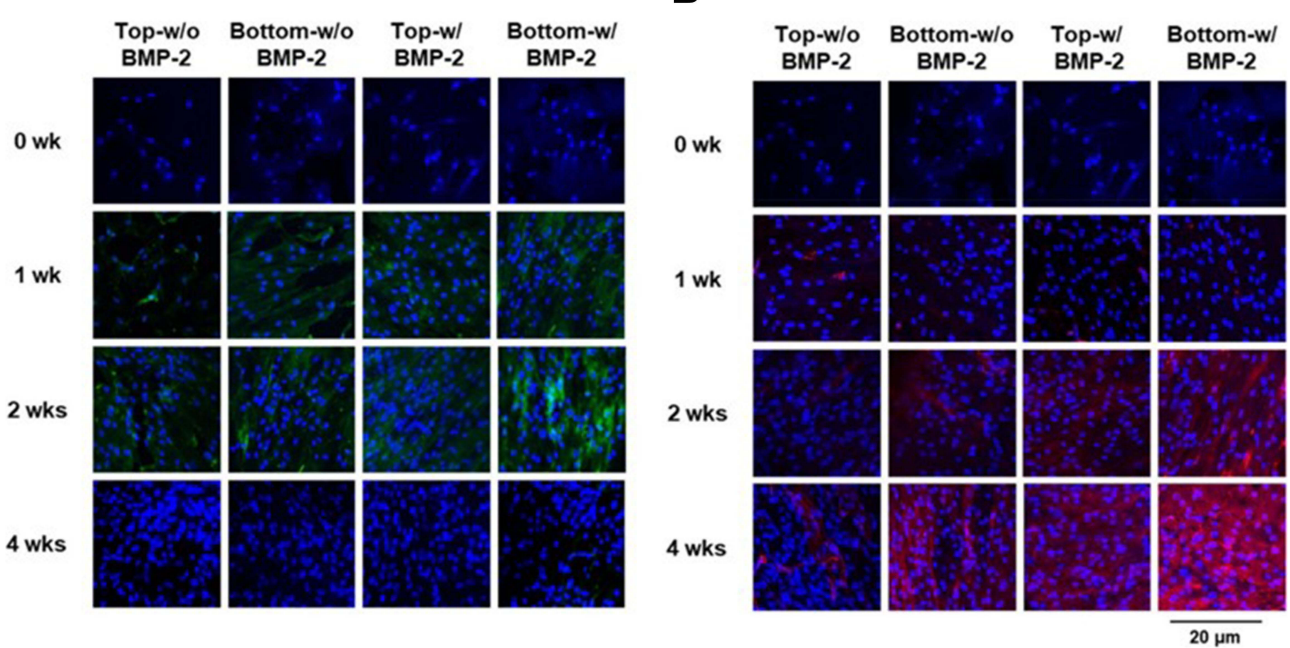

Figure 6 (A) Expression of osteogenic differentiation markers in hPDCs on Top-w/o BMP-2, Bottom-w/o BMP-2, Top-w/BMP-2, and Bottom-w/BMP-2 for 4 weeks. (A) RTPCR analysis of Runx2, type I collagen, osteopontin, and osteocalcin ( $=3$; ${ }^{*} \mathrm{p}<0.05$, $\left.{ }^{* *} \mathrm{p}<0.0 \mathrm{I}\right)$. (B) Immunocytochemical stainings of Runx2 (green color) and osteocalcin (red color) expression (blue color, cell nuclei). Reproduced with permission from Kim HY, Park JH, Byun J-H, et al. BMP-2-immobilized porous matrix with leaf-stacked structure as a bioactive GBR membrane.ACS Appl Mater Interfaces. 2018;10(36):301 I5-30124. ${ }^{151}$ Copyright (C) 2018 American Chemical Society.

be further improved. Among various growth factors, BMP-2 is often considered the most effective osteogenic growth factor. However, it should be noted that direct injection can cause symptoms such as edema and low blood pressure. A long-term and orderly release through the control of micromodels is expected to solve this limitation. A composite freeze-dried hydrogel scaffold of gelatin/heparin-coated gelatin/chitosan was prepared to administer two drugs with different release kinetics. VEGF was loaded in the outer layer for the initial release of VEGF, which induced angiogenesis in the defect area and provided blood supply. In contrast, the inner layer was loaded with BMP-4 for sustained release for sustained 
osteogenesis. ${ }^{152}$ So far, there is no ideal experimental model to satisfy most medical applications of articulation.

We can foresee that precision medicine will become a trend in the future, and highly perforated spherical models can be used in the bionics of tumor microstructures. The individual characteristic tumor markers produced by the acellular matrix of the patient's lesion tissue can be loaded with the seeded cells of the specific patient to construct a precise personal joint disease drug screening model to achieve precise medical treatment for the individual patients. As a result, medicines suitable for patients can be obtained, and individual patients can be treated accurately. However, the inability to maintain the biological activity of the characteristic tumor proteins secreted by cancer cells for a long time has become a bottleneck in the research of individualized and precise treatment of cancer patients. In addition, maintaining the supply of gradient oxygen and the delivery of vascularized nutrients are several issues that will remain unaddressed. In addition, injection therapy to stimulate the autonomous regeneration of cartilage will be applied in the joint disease treatment. Notably, the autonomous regeneration of cartilage by loading BMSCs and related active factors must be considered. However, a considerable dilemma is being faced in terms of whether it is the tumor markers of the lesion or the maintenance of the activity of the factors that assist regeneration. To a considerable extent, this problem has been solved by coating them with polymeric hydrogels based on chitosan or other natural polymers, which, however, is expecting a more effective way to solve the dilemma. The application of fast and efficient high-throughput chips in drug screening has become a new trend, but it is still a challenge to simulate the effects of different pressure stimuli and oxygen gradients in the body on tumor tissues. With further optimization, the incessant emergence of various in vitro models by considering the advantages and disadvantages of the existing strategies and resultant products will undoubtedly find their way in the future.

\section{Acknowledgments}

This study received financial support from the National Natural Science Foundation of China (NSFC, 32071323, 81971734, and 31800794), National Key R\&D Program of China (2019YFB1105600), Natural Science Foundation of Fujian Province (2019J01076), the support by the Fundamental Research Funds for the Central Universities
(ZQN-713), Funds for Foreign Experts from Ministry of Science and Technology, China (G20190013023), and Program for Innovative Research Team in Science and Technology in Fujian Province.

\section{Disclosure}

The authors declare that they have no known competing financial interests or personal relationships that could have appeared to influence the work reported in this paper.

\section{References}

1. Firestein GS. Evolving concepts of rheumatoid arthritis. Nature. 2003;423(6937):356-361. doi:10.1038/nature01661

2. Wang SS, Lv J, Meng S, et al. Recent advances in nanotheranostics for treat-to-target of rheumatoid arthritis. Adv Healthcare Mater. 2020;9(6):1901541. doi:10.1002/adhm.201901541

3. Kwon H, Brown WE, Lee CA, et al. Surgical and tissue engineering strategies for articular cartilage and meniscus repair. Nat Rev Rheumatol. 2019;15(9):550-570. doi:10.1038/s41584-019-0255-1

4. Yan W, Tu B, Liu YY, et al. Suppressive effects of plumbagin on invasion and migration of breast cancer cells via the inhibition of STAT3 signaling and down-regulation of inflammatory cytokine expressions. Bone Res. 2019;7:362-370. doi:10.4248/br201304007

5. Eftekhari A, Maleki Dizaj S, Sharifi S, et al. The use of nanomaterials in tissue engineering for cartilage regeneration; current approaches and future perspectives. Int J Mol Sci. 2020;21 (2):536. doi:10.3390/ijms21020536

6. Grogan SP, Dorthé EW, Glembotski NE, et al. Cartilage tissue engineering combining microspheroid building blocks and microneedle arrays. Connect Tissue Res. 2019;61(2):1-15. doi:10.1080/ 03008207.2019.1617280

7. Machino R, Matsumoto K, Taniguchi D, et al. Replacement of rat tracheas by layered, trachea-like, scaffold-free structures of human cells using a bio-3D printing system. Adv Healthcare Mater. 2019;8(7):1800983. doi:10.1002/adhm.201800983

8. Murata D, Kunitomi Y, Harada K, et al. Osteochondral regeneration using scaffold-free constructs of adipose tissue-derived mesenchymal stem cells made by a bio three-dimensional printer with a needle-array in rabbits. Regen Ther. 2020;15:77-89. doi:10.1016/j.reth.2020.05.004

9. Kelm JM, Timmins NE, Brown CJ, et al. Method for generation of homogeneous multicellular tumor spheroids applicable to a wide variety of cell types. Biotechnol Bioeng. 2003;83 (2):173-180. doi:10.1002/bit.10655

10. Page H, Flood P, Reynaud EG. Three-dimensional tissue cultures: current trends and beyond. Cell Tissue Res. 2013;352(1):123-131. doi:10.1007/s00441-012-1441-5

11. Grimes DR, Fletcher AG, Partridge M. Oxygen consumption dynamics in steady-state tumour models. Royal Soc Open Sci. 2014;1(1):140080. doi:10.1098/rsos. 140080

12. Monteiro CF, Santos SC, Custódio CA, et al. Human platelet lysates-based hydrogels: a novel personalized 3D platform for spheroid invasion assessment. Adv Sci. 2020;7(7):1902398. doi:10.1002/advs.201902398

13. Sit KH, Wong KP, Bay BH. An efficient and simple method of non-enzymatic detachment of monolayer cultures into single cell suspension. Methods Cell Sci. 1991;13(4):257-259. doi:10.1007/ BF02388258

14. Caron M, Emans PJ, Coolsen M, et al. Redifferentiation of dedifferentiated human articular chondrocytes: comparison of 2D and 3D cultures. Osteoarthritis Cartilage. 2012;20 (10):1170-1178. doi:10.1016/j.joca.2012.06.016 
15. Hirao M, Tamai N, Tsumaki N, Yoshikawa H, Myoui A. Oxygen tension regulates chondrocyte differentiation and function during endochondral ossification. $J$ Biol Chem. 2006;281 (41):31079-31092. doi:10.1074/jbc.M602296200

16. Tevis KM, Colson YL, Grinstaff MW. Embedded spheroids as models of the cancer microenvironment. Adv Biosyst. 2017;1 (10):1700083. doi:10.1002/adbi.201700083

17. Tamaki M, Mcdonald W, Amberger VR, et al. Implantation of C6 astrocytoma spheroid into collagen type I gels: invasive, proliferative, and enzymatic characterizations. J Neurosurg. 1997;87 (4):602-609. doi:10.3171/jns.1997.87.4.0602

18. Liu XM, Chen YH, Mao AS, et al. Molecular recognition-directed site-specific release of stem cell differentiation inducers for enhanced joint repair. Biomaterials. 2019;232:119644. doi:10.1016/j.biomaterials.2019.119644

19. Gan D, Wang Z, Xie C, et al. Mussel-inspired tough hydrogel with in situ nanohydroxyapatite mineralization for osteochondral defect repair. Adv Healthcare Mater. 2019;8(22):1901103. doi:10.1002/adhm.201901103

20. Woodell-May E, Sommerfeld SD. Role of inflammation and the immune system in the progression of osteoarthritis. J Orthop Res. 2020;38(2):253-257. doi:10.1002/jor.24457

21. Ansari MY, Ahmad N, Haqqi TM. Oxidative stress and inflammation in osteoarthritis pathogenesis: role of polyphenols. Biomed Pharmacother. 2020;129:110452. doi:10.1016/j. biopha.2020.110452

22. Conaghan PG, Cook AD, Hamilton JA, Tak PP. Therapeutic options for targeting inflammatory osteoarthritis pain. Nat Rev Rheumatol. 2019;15(6):355-363. doi:10.1038/s41584-019-0221-y

23. Francisco V, Ruiz-Fernandez C, Pino J, et al. Adipokines: linking metabolic syndrome, the immune system, and arthritic diseases. Biochem Pharmacol. 2019;165:196-206. doi:10.1016/j. bcp.2019.03.030

24. Karonitsch T, Beckmann D, Dalwigk K, et al. Targeted inhibition of Janus kinases abates interfon gamma-induced invasive behaviour of fibroblast-like synoviocytes. Rheumatology. 2018;57 (3):572-577. doi:10.1093/rheumatology/kex426

25. Bonelli M, Dalwigk K, Platzer A, et al. IRF1 is critical for the TNF-driven interferon response in rheumatoid fibroblast-like synoviocytes. Exp Mol Med. 2019;51(7):75. doi:10.1038/ s12276-019-0267-6

26. Harrell CR, Markovic BS, Fellabaum C, Arsenijevic A, Volarevic V. Mesenchymal stem cell-based therapy of osteoarthritis: current knowledge and future perspectives. Biomed Pharmacother. 2019;109:2318-2326. doi:10.1016/j.biopha.20 18.11.099

27. Broeren MGA, Waterborg CEJ, Wiegertjes $\mathrm{R}$, et al. A three-dimensional model to study human synovial pathology. Altex. 2019;36(1):18-28. doi:10.14573/altex.1804161

28. Schreiner AJ, Stoker AM, Bozynski CC, et al. Clinical application of the basic science of articular cartilage pathology and treatment. J Knee Surg. 2020;33(11):1056-1068. doi:10.1055/ s-0040-1712944

29. Andreas K, Haeupl T, Luebke C, et al. Antirheumatic drug response signatures in human chondrocytes: potential molecular targets to stimulate cartilage regeneration. Arthritis Res Ther. 2009;11(1):R15. doi:10.1186/ar2605

30. Ibold Y, Frauenschuh S, Kaps C, et al. Development of a high-throughput screening assay based on the 3-dimensional pannus model for rheumatoid arthritis. J Biomol Screen. 2007;12 (7):956-965. doi:10.1177/1087057107307147

31. Arrigoni C, Lopa S, Candrian C, Moretti M. Organs-on-A-chip as model systems for multifactorial musculoskeletal diseases. Curr Opin Biotechnol. 2020;63:79-88. doi:10.1016/j.copbio.20 19.12.006
32. Matai I, Kaur G, Seyedsalehi A, McClinton A, Laurencin CT. Progress in 3D bioprinting technology for tissue/organ regenerative engineering. Biomaterials. 2020;226:119536. doi:10.1016/j. biomaterials.2019.119536

33. Shi J, Liu S, Zhang L, et al. Smart textile-integrated microelectronic systems for wearable applications. Adv Mater. 2020;32 (5):1901958. doi:10.1002/adma.201901958

34. Zhou J, Sun J, Zhou X, et al. Resource management for improving soft-error and lifetime reliability of real-time MPSoCs. IEEE Trans Comput-Aided Des Integr Circuits Syst. 2019;38 (12):2215-2228. doi:10.1109/tcad.2018.2883993

35. Coppeta JR, Mescher MJ, Isenberg BC, et al. A portable and reconfigurable multi-organ platform for drug development with onboard microfluidic flow control. Lab Chip. 2017;17 (1):134-144. doi:10.1039/c6lc01236a

36. Frenette PS, Pinho S, Lucas D, Scheiermann C. Mesenchymal stem cell: keystone of the hematopoietic stem cell Niche and a stepping-stone for regenerative medicine. Annu Rev Immunol. 2013;31(1):285-316. doi:10.1146/annurev-immunol-032712095919

37. Ren G, Esposito M, Kang YJ. Bone metastasis and the metastatic niche. J Mol Med. 2015;93(11):1203-1212. doi:10.1007/s00109015-1329-4

38. Shen Y, Nilsson SK. Bone, microenvironment and hematopoiesis. Curr Opin Hematol. 2012;19(4):250. doi:10.1097/ MOH.0b013e328353c714

39. Morrison SJ, Scadden DT. The bone marrow niche for haematopoietic stem cells. Nature. 2014;505(7483):327-334. doi:10.1038/ nature 12984

40. Brittberg M, Lindahl A, Nilsson A, et al. Treatment of deep cartilage defects in the knee with autologous chondrocyte transplantation. $N$ Engl $J$ Med. 1994;331(14):889-895. doi:10.1056/nejm199410063311401

41. Schett G, Tohidast-Akrad M, Steiner G, et al. The stressed synovium. Arthritis Res. 2001;3(2):80-86. doi:10.1186/ar144

42. Occhetta P, Mainardi A, Votta E, et al. Hyperphysiological compression of articular cartilage induces an osteoarthritic phenotype in a cartilage-on-A-chip model. Nat Biomed Eng. 2019;3 (7):545-557. doi:10.1038/s41551-019-0406-3

43. Rosser J, Bachmann B, Jordan C, et al. Microfluidic nutrient gradient-based three-dimensional chondrocyte culture-on-A-chip as an in vitro equine arthritis model. Mater Today Bio. 2019;4:100023. doi:10.1016/j.mtbio.2019.100023

44. Pina S, Ribeiro VP, Marques CF, et al. Scaffolding strategies for tissue engineering and regenerative medicine applications. Materials. 2019;12(11):1824. doi:10.3390/ma12111824

45. Williams DF. Challenges with the development of biomaterials for sustainable tissue engineering. Front Bioeng Biotechnol. 2019;7:127. doi:10.3389/fbioe.2019.00127

46. Do Y, Nagarkatti PS, Nagarkatti M. Role of CD44 and hyaluronic acid (HA) in activation of alloreactive and antigen-specific T cells by bone marrow-derived dendritic cells. J Immunother. 2004;27 (1):1. doi:10.1097/00002371-200401000-00001

47. Tesar BM, Jiang D, Liang $\mathrm{J}$, et al. The role of hyaluronan degradation products as innate alloimmune agonists. Am $J \quad$ Transplant. 2010;6(11):2622-2635. doi:10.1111/j.16006143.2006.01537.x

48. Fan JB, Park H, Lee MK, et al. Adipose-derived stem cells and BMP-2 delivery in chitosan-based 3D constructs to enhance bone regeneration in a rat mandibular defect model. Tissue Eng Part A. 2014;20(15-16):2169-2179. doi:10.1089/ten.tea.2013.0523

49. Unagolla JM, Jayasuriya AC. Hydrogel-based 3D bioprinting: a comprehensive review on cell-laden hydrogels, bioink formulations, and future perspectives. Appl Mater Today. 2020;18:100479. doi:10.1016/j.apmt.2019.100479 
50. Islam MM, Shahruzzaman M, Biswas S, Sakib MN, Rashid TU. Chitosan based bioactive materials in tissue engineering applications-A review. Bioact Mater. 2020;5(1):164-183. doi:10.1016/j.bioactmat.2020.01.012

51. Miyazaki S, Ishii K, Nadai T. The use of chitin and chitosan as drug carriers. Chem Pharm Bull (Tokyo). 1981;29 (10):3067-3069. doi:10.1248/cpb.29.3067

52. Crompton KE, Goud JD, Bellamkonda RV, et al. Polylysinefunctionalised thermoresponsive chitosan hydrogel for neural tissue engineering. Biomaterials. 2007;28(3):441-449. doi:10.1016/ j.biomaterials.2006.08.044

53. Wei YN, Wang QQ, Gao TT, et al. 3-D culture of human umbilical vein endothelial cells with reversible thermosensitive hydroxybutyl chitosan hydrogel. J Mater Sci Mater Med. 2013;24 (7):1781-1787. doi:10.1007/s10856-013-4918-1

54. Stüdle C, Martin QV, Haumer A, Guerrero J, Martin IJB. Spatially confined induction of endochondral ossification by functionalized hydrogels for ectopic engineering of osteochondral tissues. Biomaterials. 2018;171:219-229. doi:10.1016/j. biomaterials.2018.04.025

55. Muzzarelli RA, Biagini G, Bellardini M, Simonelli L, Castaldini C, Fratto G. Osteoconduction exerted by methylpyrrolidinone chitosan used in dental surgery. Biomaterials. 1993;14 (1):39-43. doi:10.1016/0142-9612(93)90073-b

56. Cumming $\mathrm{MH}$, Hall $\mathrm{B}$, Hofman $\mathrm{K}$. Isolation and characterisation of major and minor collagens from Hyaline cartilage of Hoki (Macruronus novaezelandiae). Mar Drugs. 2019;17(4):223. doi:10.3390/md17040223

57. Cardwell RD, Dahlgren LA, Goldstein AS. Electrospun fiber diameter, not alignment, affects mesenchymal stem cell differentiation into the tendon/ligament lineage. J Tissue Eng Regen Med. 2014;8(12):937-945. doi:10.1002/term.1589

58. Yunoki S, Hatayama H, Ebisawa M, et al. A novel fabrication method to create a thick collagen bundle composed of uniaxially aligned fibrils: an essential technology for the development of artificial tendon/ligament matrices. J Biomed Mater Res A. 2015;103(9):3054-3065. doi:10.1002/jbm.a.35440

59. Puetzer JL, Ma TC, Sallent I, Gelmi A, Stevens MM. Driving hierarchical collagen fiber formation for functional tendon, ligament, and meniscus replacement. Biomaterials. 2021;269:120527. doi:10.1016/j.biomaterials.2020.120527

60. Ma L. Collagen/chitosan porous scaffolds with improved biostability for skin tissue engineering. Biomaterials. 2003;24 (26):4833-4841. doi:10.1016/S0142-9612(03)00374-0

61. Rho KS, Jeong L, Lee G, et al. Electrospinning of collagen nanofibers: effects on the behavior of normal human keratinocytes and early-stage wound healing. Biomaterials. 2006;27 (8):1452-1461. doi:10.1016/j.biomaterials.2005.08.004

62. Chen H, Zhang Y, Ding P, et al. BMSCs encapsulated in functionalized gellan gum/collagen hydrogel for effective vascularization. ACS Appl Bio Mater. 2018;1(5):1408-1415. doi:10.1021/acsabm.8b00361

63. Seo J, Shin J-Y, Leijten J, et al. Interconnectable dynamic compression bioreactors for combinatorial screening of cell mechanobiology in three dimensions. ACS Appl Mater Interfaces. 2018;10(16):13293-13303. doi:10.1021/acsami.7b17991

64. Bersini S, Jeon JS, Dubini G, et al. A microfluidic 3D in vitro model for specificity of breast cancer metastasis to bone. Biomaterials. 2014;35(8):2454-2461. doi:10.1016/j.biomate rials.2013.11.050

65. Galuzzi M, Perteghella S, Antonioli B, et al. Human engineered cartilage and decellularized matrix as an alternative to animal osteoarthritis model. Polymers. 2018;10(7):30960663. doi:10.3 390/polym 10070738
66. Li Y, Xiao Y, Liu C. The horizon of materiobiology: a perspective on material-guided cell behaviors and tissue engineering. Chem Rev. 2017;117(5):4376-4421. doi:10.1021/acs.chemrev.6b00654

67. Kumar S. Cellular mechanotransduction: stiffness does matter. Nat Mater. 2014;13(10):918-920. doi:10.1038/nmat4094

68. Klotz BJ, Gawlitta D, Rosenberg A, Malda J, Melchels F. Gelatin-methacryloyl hydrogels: towards biofabrication-based tissue repair. Trends Biotechnol. 2016;34(5):394-407. doi:10.1016/j. tibtech.2016.01.002

69. Highley CB, Prestwich GD, Burdick JA. Recent advances in hyaluronic acid hydrogels for biomedical applications. Curr Opin Biotechnol. 2016;40:35-40. doi:10.1016/j.copbio.20 16.02.008

70. Garreta E, Oria R, Tarantino C, et al. Tissue engineering by decellularization and 3D bioprinting. Mater Today. 2017;20 (4):166-178. doi:10.1016/j.mattod.2016.12.005

71. Hubmacher D, Apte SS. The biology of the extracellular matrix: novel insights. Curr Opin Rheumatol. 2013;25(1):65-70. doi:10.1097/BOR.0b013e32835b137b

72. Lu P, Takai K, Weaver VM, et al. Extracellular matrix degradation and remodeling in development and disease. Cold Spring Harb Perspect Biol. 2011;3(12):21917992. doi:10.1101/cshperspect.a005058

73. De Santis MM, Alsafadi HN, Tas S, et al. Extracellular-matrixreinforced bioinks for 3D bioprinting human tissue. Adv Mater. 2021;33(3):2005476. doi:10.1002/adma.202005476

74. Elder BD, Eleswarapu SV, Athanasiou KA. Extraction techniques for the decellularization of tissue engineered articular cartilage constructs. Biomaterials. 2009;30(22):3749-3756. doi:10.1016/j. biomaterials.2009.03.050

75. Deng M, Tan J, Hu C, et al. Modification of PLGA Scaffold by MSC-derived extracellular matrix combats macrophage inflammation to initiate bone regeneration via TGF- $\beta$-induced protein. Adv Healthcare Mater. 2020;9(13):e2000353. doi:10.1002/ adhm.202000353

76. Chae S, Lee SS, Choi YJ, Hong DH, Cho DW. 3D cell-printing of biocompatible and functional meniscus constructs using meniscus-derived. Biomaterials. 2021;267:120466. doi:10.1016/j. biomaterials.2020.120466

77. Qiu P, Li M, Chen K, et al. Periosteal matrix-derived hydrogel promotes bone repair through an early immune regulation coupled with enhanced angio- and osteogenesis. Biomaterials. 2020;227:119552. doi:10.1016/j.biomaterials.2019.119552

78. Feng X, Hou X, Cui C, Sun S, Zhou F. Mechanical and antibacterial properties of tannic acid-encapsulated carboxymethyl chitosan/polyvinyl alcohol hydrogels. Eng Regen Med. 2021;2:57-62. doi:10.1016/j.engreg.2021.05.002

79. Dai C, Li Y, Pan W, et al. Three-dimensional high-porosity chitosan/honeycomb porous carbon/hydroxyapatite scaffold with enhanced osteoinductivity for bone regeneration. ACS Biomater Sci Eng. 2020;6(1):575-586. doi:10.1021/acsbiomaterials.9 b01381

80. Zhang X, Yin X, Luo J, et al. Novel hierarchical nitrogen-doped multiwalled carbon nanotubes/cellulose/nanohydroxyapatite nanocomposite as an osteoinductive Scaffold for enhancing bone regeneration. ACS Biomater Sci Eng. 2019;5(1):294-307. doi:10.1021/acsbiomaterials.8b00908

81. Go G, Han J, Zhen J, et al. A magnetically actuated Microscaffold containing mesenchymal stem cells for articular cartilage repair. Adv Healthcare Mater. 2017;6(13):1601378. doi:10.1002/ adhm.201601378

82. Go G, Jeong SG, Yoo A, et al. Human adipose-derived mesenchymal stem cell-based medical microrobot system for knee cartilage regeneration in vivo. Sci Robot. 2020;5(38):eaay6626. doi:10.1126/scirobotics.aay6626 
83. Steinmetz NJ, Aisenbrey EA, Westbrook KK, et al. Mechanical loading regulates human MSC differentiation in a multi-layer hydrogel for osteochondral tissue engineering. Acta Biomater. 2015;21(15):142-153. doi:10.1016/j.actbio.2015.04.015

84. Duval K, Grover H, Han L-H, et al. Modeling physiological events in 2D vs 3D cell culture. Physiology. 2017;32 (4):266-277. doi:10.1152/physiol.00036.2016

85. Mandal BB, Kundu SC. Cell proliferation and migration in silk fibroin 3D scaffolds. Biomaterials. 2009;30(15):2956-2965. doi:10.1016/j.biomaterials.2009.02.006

86. Cui L, Chen J, Yan C, Xiong D. Articular cartilage inspired the construction of LTi-DA-PVA composite structure with excellent surface wettability and low friction performance. Tribol Lett. 2021;69(2):41. doi:10.1007/s11249-021-01416-y

87. Wei W, Ma Y, Yao X, et al. Advanced hydrogels for the repair of cartilage defects and regeneration. Bioact Mater. 2021;6 (4):998-1011. doi:10.1016/j.bioactmat.2020.09.030

88. Zhang Y, Yu J, Ren K, Zuo J, Ding J, Chen X. Thermosensitive hydrogels as Scaffolds for cartilage tissue engineering. Biomacromolecules. 2019;20(4):1478-1492. doi:10.1021/acs. biomac. 9 b00043

89. Dey P, Schneider T, Chiappisi L, et al. Mimicking of chondrocyte microenvironment using in situ forming dendritic polyglycerol sulfate-based synthetic polyanionic hydrogels. Macromol Biosci. 2016;16(4):580-590. doi:10.1002/mabi.201500377

90. Inagaki Y, Kitamura N, Kurokawa T, et al. Effects of culture on PAMPS/PDMAAm double-network gel on chondrogenic differentiation of mouse C3H10T1/2 cells: in vitro experimental study. BMC Musculoskelet Disord. 2014;15(1):1-7. doi:10.1186/14712474-15-320

91. Laranga R, Duchi S, Ibrahim T, et al. Trends in bone metastasis modeling. Cancers. 2020;12(8):2315. doi:10.3390/cancers12 082315

92. Mara C, Sartori AR, Duarte AS, et al. Periosteum as a source of mesenchymal stem cells: the effects of TGF-beta 3 on chondrogenesis. Clinics. 2011;66(3):487-492. doi:10.1590/ s1807-59322011000300022

93. Zeng L, Yao Y, Wang DA, et al. Effect of microcavitary alginate hydrogel with different pore sizes on chondrocyte culture for cartilage tissue engineering. Mater Sci Eng C Mater Biol Appl. 2014;34(1):168-175. doi:10.1016/j.msec.2013.09.003

94. Remya NS, Nair PD. Engineering cartilage tissue interfaces using a natural glycosaminoglycan hydrogel matrix - an in vitro study. Mater Sci Eng C Mater Biol Appl. 2013;33(2):575-582. doi:10.1016/j.msec.2012.09.015

95. An H, Lee JW, Lee HJ, et al. Hyaluronate-alginate hybrid hydrogels modified with biomimetic peptides for controlling the chondrocyte phenotype. Carbohydr Polym. 2018;197:422-430. doi:10.1016/j.carbpol.2018.06.016

96. Nii T, Makino K, Tabata Y. Three-dimensional culture system of cancer cells combined with biomaterials for drug screening. Cancers. 2020;12(10):2754. doi:10.3390/cancers 12 102754

97. Ivanovska J, Zehnder T, Lennert P, et al. Biofabrication of 3D alginate-based hydrogel for cancer research: comparison of cell spreading, viability, and adhesion characteristics of colorectal HCT116 tumor cells. Tissue Eng Part C Methods. 2016;22 (7):708-715. doi:10.1089/ten.tec.2015.0452

98. Qiao Y, Liu X, Zhou X, et al. Gelatin templated polypeptide cocross-linked hydrogel for bone regeneration. Adv Healthcare Mater. 2020;9(1):1901239. doi:10.1002/adhm.201901239

99. Xia B, Krutkramelis K, Oakey J. Oxygen-purged microfluidic device to enhance cell viability in photopolymerized PEG hydrogel microparticles. Biomacromolecules. 2016;17(7):2459-2465. doi:10.1021/acs.biomac.6b00597
100. Ratheesh G, Vaquette C, Xiao Y, et al. Patient-specific bone particles bioprinting for bone tissue engineering. Adv Healthcare Mater. 2020;9(23):2001323. doi:10.1002/adhm.202001323

101. Kankala RK, Zhao J, Liu CG, et al. Highly porous microcarriers for minimally invasive in situ skeletal muscle cell delivery. Small. 2019;15(25):1901397. doi:10.1002/smll.201901397

102. Rothbauer M, Hoell G, Eilenberger C, et al. Monitoring tissue-level remodelling during inflammatory arthritis using a three-dimensional synovium-on-A-chip with non-invasive light scattering biosensing. Lab Chip. 2020;20(8):1461-1471. doi:10.1039/c9lc01097a

103. Mestres G, Perez RA, D'Elía NL, et al. Advantages of microfluidic systems for studying cell-biomaterial interactions-focus on bone regeneration applications. Biomed Phys Eng Express. 2019;5 (3):032001. doi:10.1088/2057-1976/ab1033

104. Wang Y, Kankala RK, Zhang J, et al. Modeling endothelialized hepatic tumor microtissues for drug screening. Adv Sci. 2020;7 (21):2002002. doi:10.1002/advs.202002002

105. Wang Y, Kankala RK, Cai -Y-Y, et al. Minimally invasive co-injection of modular micro-muscular and micro-vascular tissues improves in situ skeletal muscle regeneration. Biomaterials. 2021;277:121072. doi:10.1016/j.biomaterials.2021.121072

106. Ying W, Xuan H, Kankala RK, et al. Endothelialized microrods for minimally invasive in situ neovascularization. Biofabrication. 2020;12(1):015011. doi:10.1088/1758-5090/ab47eb

107. Gao RZ, Marriott K, Dickerson CR, et al. Design and preliminary implementation of an air microfluidics enabled soft robotic knee brace towards the management of osteoarthritis. 42nd Annual International Conferences of the Ieee Engineering in Medicine and Biology Society: Enabling Innovative Technologies for Global Healthcare Embc'20; New York: IEEE; 2020:4502-4505. doi:10.1109/EMBC44109.2020.9175677.

108. Gu Z, Fu J, Lin H, et al. Development of 3D bioprinting: from printing methods to biomedical applications. Asian J Pharm Sci. 2020;15(5):529-557. doi:10.1016/j.ajps.2019.11.003

109. De Mori A, Fernandez MP, Blunn G, Tozzi G, Roldo M. 3D printing and electrospinning of composite hydrogels for cartilage and bone tissue engineering. Polymers. 2018;10(3):285. doi:10.3390/polym10030285

110. Vanaei S, Parizi MS, Vanaei S, Salemizadehparizi F, Vanaei HR. An overview on materials and techniques in 3D bioprinting toward biomedical application. Eng Regen Med. 2021;2:1-18. doi:10.1016/j.engreg.2020.12.001

111. Wu Y, Kennedy P, Bonazza N, Yu Y, Dhawan A, Ozbolat I. Threedimensional bioprinting of articular cartilage: a systematic review. Cartilage. 2021;12(1):76-92. doi:10.1177/1947603518809410

112. Hong H, Seo YB, Kim DY, et al. Digital light processing 3D printed silk fibroin hydrogel for cartilage tissue engineering. Biomaterials. 2019;232:119679. doi:10.1016/j.biomaterials.2019.119679

113. Qiao Z, Lian M, Han Y, et al. Bioinspired stratified electrowritten fiber-reinforced hydrogel constructs with layer-specific induction capacity for functional osteochondral regeneration. Biomaterials. 2021;266(6338):120385. doi:10.1016/j.biomaterials.2020.120385

114. Wu Y, Ayan B, Moncal KK, et al. Hybrid bioprinting of zonally stratified human articular cartilage using Scaffold-free tissue strands as building blocks. Adv Healthcare Mater. 2020;9 (22):2001657. doi:10.1002/adhm.202001657

115. Xia H, Zhao D, Zhu H, et al. Lyophilized Scaffolds fabricated from 3D-printed photocurable natural hydrogel for cartilage regeneration. ACS Appl Mater Interfaces. 2018;10 (37):31704-31715. doi:10.1021/acsami.8b10926

116. de Melo BA, Jodat YA, Mehrotra S, et al. 3D printed cartilagelike tissue constructs with spatially controlled mechanical properties. Adv Funct Mater. 2019;29(51):1906330. doi:10.1002/ adfm. 201906330 
117. Zhang M, Lin R, Wang X, Xue J, Deng C. 3D printing of Haversian bone-mimicking scaffolds for multicellular delivery in bone regeneration. Sci Adv. 2020;6(12):eaaz6725. doi:10.1126/sciadv.aaz6725

118. Bai C, Ji M, Bouakaz A, Zong Y, Wan M. Design and characterization of an acoustically and structurally matched 3-D-printed model for transcranial ultrasound imaging. IEEE Trans Ultrason Ferroelectr Freq Control. 2018;65(5):741-748. doi:10.1109/ tuffc. 2018.2811756

119. Han X, Sun M, Chen B, et al. Lotus seedpod-inspired internal vascularized 3D printed scaffold for bone tissue repair. Bioact Mater. 2021;6(6):1639-1652. doi:10.1016/j.bioactmat.20 20.11.019

120. Moncal KK, Heo DN, Godzik KP, et al. 3D printing of poly (epsilon-caprolactone)/poly(D,L-lactide-co- glycolide)/hydroxyapatite composite constructs for bone tissue engineering. $J$ Mater Res. 2018;33(14):1972-1986. doi:10.1557/jmr.2018.111

121. Rukavina P, Koch F, Wehrle M, et al. In vivo evaluation of bioprinted prevascularized bone tissue. Biotechnol Bioeng. 2020;117(12):3902-3911. doi:10.1002/bit.27527

122. Zhao Y, Hou Y, Li Z, Wang Z, Yan X. Powder-based 3D printed porous structure and its application as bone Scaffold. Front Mater. 2020;7:150. doi:10.3389/fmats.2020.00150

123. Diloksumpan $\mathrm{P}$, Ruijter $\mathrm{MD}$, Castilho $\mathrm{M}$, et al. Combining multi-scale 3D printing technologies to engineer reinforced hydrogel-ceramic interfaces. Biofabrication. 2020;12(2):025014. doi:10.1088/1758-5090/ab69d9

124. Jin L, Wang $\mathrm{T}$, Zhu ML, et al. Electrospun fibers and tissue engineering. J Biomed Nanotechnol. 2012;8(1):1-9. doi:10.1166/ jbn.2012.1360

125. Megelski S, Stephens JS, Chase DB, Rabolt JF. Micro- and nanostructured surface morphology on electrospun polymer fibers. Macromolecules. 2002;35(22):8456-8466. doi:10.1021/ ma020444a

126. Sun J, Vijayavenkataraman S, Liu H. An overview of scaffold design and fabrication technology for engineered knee Meniscus. Materials. 2017;10(1):29. doi:10.3390/ma10010029

127. Chen WM, Xu Y, Li YQ, et al. 3D printing electrospinning fiber-reinforced decellularized extracellular matrix for cartilage regeneration. Chem Eng J. 2020;382:122986. doi:10.1016/j. cej.2019.122986

128. Wu L, Gu Y, Liu L, et al. Hierarchical micro/nanofibrous membranes of sustained releasing VEGF for periosteal regeneration. Biomaterials. 2020;227:119555. doi:10.1016/j.biomaterials.20 19.119555

129. Shalumon KT, Lai GJ, Chen $\mathrm{CH}$, et al. Modulation of Bonespecific tissue regeneration by incorporating bone morphogenetic protein and controlling the shell thickness of silk fibroin/chitosan/ nanohydroxyapatite core-shell nanofibrous membranes. ACS Appl Mater Interfaces. 2015;7(38):21170. doi:10.1021/ acsami.5b04962

130. Ma Z, He W, Yong T, et al. Grafting of gelatin on electrospun Poly(caprolactone) nanofibers to improve endothelial cell spreading and proliferation and to control cell orientation. Tissue Eng. 2005;11(7-8):1149-1158. doi:10.1089/ten.2005.11.1149

131. Colunga T, Dalton S. Building blood vessels with vascular progenitor cells. Trends Mol Med. 2018;24(7):630-641. doi:10.1016/ j.molmed.2018.05.002

132. Kim JW, Han YS, Lee HM, Kim JK, Kim YJ. Effect of morphological characteristics and biomineralization of 3D-printed gelatin/hyaluronic acid/hydroxyapatite composite scaffolds on bone tissue regeneration. Int J Mol Sci. 2021;22(13):6794. doi:10.3390/ ijms 22136794

133. Zhang J, Ji Y, Jiang S, et al. Calcium-collagen coupling is vital for biomineralization schedule. Adv Sci. 2021;8(15):2100363. doi:10.1002/advs.202100363
134. Cheng L, Chen Z, Cai Z, et al. Bioinspired functional black phosphorus electrospun fibers achieving recruitment and biomineralization for staged bone regeneration. Small. 2020;16 (50):2005433. doi:10.1002/smll.202005433

135. Zhang J, Tang L, Qi H, Zhao Q, Liu Y, Zhang Y. Dual function of magnesium in bone biomineralization. Adv Healthcare Mater. 2019;8(21):1901030. doi:10.1002/adhm.201901030

136. Damerau A, Pfeiffenberger M, Weber M-C, et al. A human osteochondral tissue model mimicking cytokine-induced key features of arthritis in vitro. Int J Mol Sci. 2020;22(1):128. doi:10.3390/ijms 22010128

137. Jana S, Singh SK. Identification of selective MMP-9 inhibitors through multiple e-pharmacophore, ligand-based pharmacophore, molecular docking, and density functional theory approaches. $J$ Biomol Struct Dyn. 2019;37(4):944-965. doi:10.1080/ 07391102.2018.1444510

138. Ria F, Pirolli D, Di Sante G, et al. Selective inhibitors of T cell receptor recognition of antigen-MHC Complexes for Rheumatoid Arthritis. ACS Med Chem Lett. 2019;10(4):644-649. doi:10.1021/ acsmedchemlett.8b00601

139. Wang J, Peng W, Li X, et al. Towards to potential 2-cyanopyrimidines cathepsin- $\mathrm{K}$ inhibitors: an in silico design and screening research based on comprehensive application of quantitative structure-activity relationships, molecular docking and ADMET prediction. $J$ Mol Struct. 2019;1195:914-928. doi:10.1016/j.molstruc.2019.06.020

140. Monteiro MM, Gaspar VM, Ferreira LP, et al. Hydrogel 3D in vitro tumor models for screening cell aggregation mediated drug response. Biomater Sci. 2020;8(7):1855-1864. doi:10.1039/ c9bm02075f

141. Jorgensen C, Simon M. In vitro human joint models combining advanced 3D cell culture and cutting-edge 3D bioprinting technologies. Cells. 2021;10(3):596. doi:10.3390/cells10030596

142. Liu C, Xu X, Cui W, et al. Metal-organic framework (MOF)-based biomaterials in bone tissue engineering. Eng Regen Med. 2021;2:105-108. doi:10.1016/j.engreg.2021.09.001

143. Luo J, Zhang X, Machuki JO, et al. Three-dimensionally N-Doped GrapheneHydroxyapatite/Agarose 2 as an osteoinductive Scaffold for enhancing bone regeneration. ACS Appl Bio Mater. 2019;2(1):299-310. doi:10.1021/acsabm.8b00599

144. Jin Z, Ren J, Qi S. Human bone mesenchymal stem cells-derived exosomes overexpressing microRNA-26a-5p alleviate osteoarthritis via down-regulation of PTGS2. Int Immunopharmacol. 2020;78:105946. doi:10.1016/j.intimp.2019.105946

145. Wu J, Kuang L, Chen C, et al. miR-100-5p-abundant exosomes derived from infrapatellar fat pad MSCs protect articular cartilage and ameliorate gait abnormalities via inhibition of mTOR in osteoarthritis. Biomaterials. 2019;206:87-100. doi:10.1016/j. biomaterials.2019.03.022

146. Zhang S, Chuah SJ, Lai RC, Hui JHP, Lim SK, Toh WS. MSC exosomes mediate cartilage repair by enhancing proliferation, attenuating apoptosis and modulating immune reactivity. Biomaterials. 2018;156:16-27. doi:10.1016/j.biomaterials.20 17.11.028

147. Dahlin RL, Kinard LA, Lam J, et al. Articular chondrocytes and mesenchymal stem cells seeded on biodegradable scaffolds for the repair of cartilage in a rat osteochondral defect model. Biomaterials. 2014;35(26):7460-7469. doi:10.1016/j.biomaterials.2014.05.055

148. Gamer LW, Pregizer S, Gamer J, et al. The role of Bmp2 in the maturation and maintenance of the murine knee joint. $\mathrm{Am} \mathrm{Soc}$ Bone Miner Res. 2018;33(9):1708-1717. doi:10.1002/jbmr.3441

149. Lee C, Hwang HS, Kim S, Fan J, Min L. Inspired by nature: facile design of nanoclay-organic hydrogel bone sealant with multifunctional properties for robust bone regeneration. $A d v$ Funct Mater. 2020;30(43):2003717. doi:10.1002/adfm.2 02003717 
150. Weihe E, Nohr D, Schäfer MK-H. Calcitonin gene related peptide gene expression in collagen-induced arthritis. Can J Physiol Pharmacol. 1995;73(7):1015. doi:10.1139/y95-142

151. Kim HY, Park JH, Byun J-H, et al. BMP-2-immobilized porous matrix with leaf-stacked structure as a bioactive GBR membrane. ACS Appl Mater Interfaces. 2018;10(36):30115-30124. doi:10.1021/acsami.8b09558

152. Lee SS, Kim JH, Jeong J, et al. Sequential growth factor releasing double cryogel system for enhanced bone regeneration. Biomaterials. 2020;257:120223. doi:10.1016/j.biomaterials.20 20.120223

153. Jeong SH, Kim M, Kim TY, et al. Supramolecular injectable hyaluronate hydrogels for cartilage tissue regeneration. ACS Appl Bio Mater. 2020;3(8):5040-5047. doi:10.1021/acsabm.0c 00537

154. Belleghem SV, Torres L, Santoro M, Mahadik B, Fisher JP. Hybrid 3D printing of synthetic and cell-laden bioinks for shape retaining soft tissue grafts. Adv Funct Mater. 2020;30 (3):1907145. doi:10.1002/adfm.201907145

155. Chen PF, Zheng L, Wang YY, et al. Desktop-stereolithography 3D printing of a radially oriented extracellular matrix/mesenchymal stem cell exosome bioink for osteochondral defect regeneration. Theranostics. 2019;9(9):2439-2459. doi:10.7150/ thno.31017

156. Lai YX, Li Y, Cao HJ, et al. Osteogenic magnesium incorporated into PLGA/TCP porous scaffold by 3D printing for repairing challenging bone defect. Biomaterials. 2019;197:207-219. doi:10.1016/j.biomaterials.2019.01.013
157. Shuai CJ, Yang WJ, Feng P, Peng SP, Pan H. Accelerated degradation of HAP/PLLA bone scaffold by PGA blending facilitates bioactivity and osteoconductivity. Bioact Mater. 2021;6 (2):490-502. doi:10.1016/j.bioactmat.2020.09.001

158. Cui X, Soliman BG, Alcala-Orozco C, et al. Rapid photocrosslinking of silk hydrogels with high cell density and enhanced shape fidelity. Adv Healthcare Mater. 2020;9(4):1901667. doi:10.1002/adhm.201901667

159. Song KH, Heo SJ, Peredo AP. Influence of fiber stiffness on meniscal cell migration into dense fibrous networks. Adv Healthcare Mater. 2020;9(8):e1901228. doi:10.1002/adhm.201 901228

160. Hu H, Dong L, Bu Z, et al. miR-23a-3p-abundant small extracellular vesicles released from $\mathrm{G}$ elma/nanoclay hydrogel for cartilage regeneration. J Extracell Vesicles. 2020;9(1):1778883. doi:10.1080/20013078.2020.1778883

161. Qiao YS, Liu XZ, Zhou XC, et al. Gelatin templated polypeptide co-cross-linked hydrogel for bone regeneration. Adv Healthcare Mater. 2020;9(1):e1901239. doi:10.1002/adhm.201901239

162. Jeon JS, Bersini S, Gilardi M, et al. Human 3D vascularized organotypic microfluidic assays to study breast cancer cell extravasation. Proc Natl Acad Sci U S A. 2015;112(7):E818E18. doi:10.1073/pnas.1501426112

163. Singh YP, Moses JC, Bhardwaj N, et al. Overcoming the dependence on animal models for osteoarthritis therapeutics - the promises and prospects of in vitro models. Adv Healthcare Mater. 2021;10(20):2100961. doi:10.1002/adhm.202100961
Drug Design, Development and Therapy

\section{Publish your work in this journal}

Drug Design, Development and Therapy is an international, peerreviewed open-access journal that spans the spectrum of drug design and development through to clinical applications. Clinical outcomes, patient safety, and programs for the development and effective, safe, and sustained use of medicines are a feature of the journal, which has also

\section{Dovepress}

been accepted for indexing on PubMed Central. The manuscript management system is completely online and includes a very quick and fair peer-review system, which is all easy to use. Visit http://www. dovepress.com/testimonials.php to read real quotes from published authors. 